\title{
Cyclovirobuxine D inhibits colorectal cancer tumorigenesis via the CTHRC1-AKT/ERK-Snail signaling pathway
}

\author{
FENGQI JIANG ${ }^{1}$, YAODONG CHEN ${ }^{2}$, SHUO REN $^{1}$, ZIZHUO LI $^{1}, \mathrm{KAN} \mathrm{SUN}^{3}$, \\ YANWEI XING ${ }^{1}$, YUEKUN ZHU ${ }^{1}$ and DAXUN PIAO ${ }^{1}$
${ }^{2}$ Department of Ultrasonic Imaging, First Clinical Medical College, Shanxi Medical University, Taiyuan, Shanxi 030001; ${ }^{3}$ Department of General Surgery, Heilongjiang Provincial Hospital, Harbin, Heilongjiang 150001, P.R. China \\ ${ }^{1}$ Department of Colorectal Surgery, The First Affiliated Hospital of Harbin Medical University, Harbin, Heilongjiang 150001;
}

Received September 25, 2019; Accepted March 13, 2020

DOI: $10.3892 /$ ijo.2020.5038

\begin{abstract}
Cyclovirobuxine D (CVB-D) is an alkaloid, which is mainly derived from Buxus microphylla. It has been reported that CVB-D has positive effects on breast cancer, gastric cancer and other malignant tumors. However, to the best of our knowledge, there are no reports regarding the effects of CVB-D on colorectal cancer (CRC). The purpose of the present study was to determine the anticancer effects of CVB-D and further elucidate its molecular mechanism(s). DLD-1 and LoVo cell lines were selected to evaluate the antitumor effect of CVB-D. Cytotoxicity, viability and proliferation were evaluated by the MTT and colony formation assays. Flow cytometry was used to detect the effects on apoptosis and the cell cycle in CVB-D-treated CRC cells. The migration and invasion abilities of CRC cells were examined by wound healing and Transwell assays. In addition, RNA sequencing, bioinformatics analysis and western blotting were performed to investigate the target of drug action and clarify the molecular mechanisms. A xenograft model was established using nude mice, and ultrasound was employed to assess the preclinical therapeutic effects of CVB-D in vivo. It was identified that CVB-D inhibited the proliferation, migration,
\end{abstract}

Correspondence to: Professor Daxun Piao, Department of Colorectal Surgery, The First Affiliated Hospital of Harbin Medical University, 23 Youzheng Street, Harbin, Heilongjiang 150001, P.R. China

E-mail: piaodaxun@sina.com

Abbreviations: CVB-D, cyclovirobuxine D; CTHRC1, collagen triple helix repeat containing 1; COAD, colon adenocarcinoma; CRC, colorectal cancer; NPCs, natural plant compounds; EMT, epithelial-mesenchymal transition; DEGs, differentially expressed genes; OS, overall survival; DFS, disease-free survival; CPA, color power angiography; CFI, color Doppler flow imaging; USE, ultrasonic elastosonography

Key words: cyclovirobuxine D, colorectal cancer, collagen triple helix repeat containing 1-AKT/ERK-Snail signaling pathway, epithelial-to-mesenchymal transition stemness, angiogenesis and epithelial-mesenchymal transition of CRC cells, and induced apoptosis and S-phase arrest. In addition, CVB-D significantly inhibited the growth of xenografts. It is notable that CVB-D exerted anticancer effects in CRC cells partly by targeting collagen triple helix repeat containing 1 (CTHRC1), which may be upstream of the AKT and ERK pathways. CVB-D exerted anticancer effects through the CTHRC1-AKT/ERK-Snail signaling pathway. Targeted therapy combining CTHRC1 with CVB-D may offer a promising novel therapeutic approach for CRC treatment.

\section{Introduction}

Colorectal cancer (CRC), including colon and rectal cancer, is one of the most common types of gastrointestinal malignant tumors, ranking third in males and second in females, and poses a serious threat to human health (1). Colon adenocarcinoma (COAD) is the most common histopathological type of $\mathrm{CRC}$, and COAD morbidity and mortality rates are increasing. In 2018, the number of new cases of COAD surpassed 1 million and $>500,000$ COAD patients succumbed to the disease (2). Therefore, studying the pathogenesis of and treatments for COAD are particularly important (3). Significant breakthroughs have been made in the early diagnosis and treatment of CRC in recent decades (4). Unfortunately, the overall survival (OS) rate of patients with $\mathrm{CRC}$ remains poor, especially in the advanced stage, mainly due to recurrence and metastasis (5).

Accumulating evidence has shown that natural plant compounds (NPCs), such as curcumin, resveratrol and dictamnine, have significant clinical benefits in cancer, such as inhibiting cell proliferation, affecting the cell cycle, and promoting apoptosis and/or autophagy (6-8). In addition, bioactive natural components extracted from plants have received attention for their inhibition of epithelial-mesenchymal transformation (EMT) through anti-inflammatory, anti-fibrosis or anti-oxidant mechanisms (9). It has been demonstrated that pathological EMT is inhibited by NPCs via different cellular signal transduction pathways (10).

Cyclovirobuxine D (CVB-D; Chemical Abstracts Service number: 860-79-7; $\mathrm{C}_{26} \mathrm{H}_{46} \mathrm{~N}_{2} \mathrm{O}$; molecular weight: 402.66) is an alkaloid, which is mainly derived from Buxus microphylla. 
B. microphylla is a Traditional Chinese Medicine with a long history of treating cardiovascular diseases and was recorded hundreds of years ago in the Compendium of Materia (11). Previous studies have indicated that CVB-D can induce autophagy-related death in human breast cancer cells via the AKT/mammalian target of rapamycin (mTOR) pathway (12), and induce mitochondria-mediated apoptosis, thereby inhibiting the proliferation of gastric cancer cells (13). However, to the best of our knowledge, there are no reports on the effects of CVB-D in CRC.

The metastasis of malignant tumors is the main characteristic of the EMT. EMT is a complex biological process, in which epithelial cells undergo multiple morphological and biochemical changes and genetic rearrangements, leading to a mesenchymal-like phenotype (14-16). Enhanced mesenchymal phenotypes alter a series of biological behaviors of cancer cells, such as proliferation, stemness, anti-apoptosis, migration, invasion and radio-/chemo-sensitivity (17). Therefore, targeting EMT is a powerful approach for inhibiting the metastasis of malignant tumors. A group of transcription factors, including Snail, Slug, Twist, zinc finger E-box-binding homeobox (ZEB)1 and ZEB2, also known as EMT regulators, directly or indirectly inhibit the activity of the E-cadherin promoter and precisely orchestrate the EMT process, leading to expression of the mesenchymal markers $\mathrm{N}$-cadherin and vimentin (18). In addition, a number of oncogenes and tumor suppressor genes are involved in the EMT process as upstream or downstream factors (19). One of these proteins, collagen triple helix repeat containing 1 (CTHRC1), was first found to be involved in vascular remodeling and plays a key role in the response of cells to arterial injury (20). CTHRC1 is overexpressed in numerous solid tumors and plays an important role in tumorigenesis and metastasis, particularly in CRC $(21,22)$, gastric cancer, melanoma, oral cancer, pancreatic cancer and hepatocellular cancer (23-27). CTHRC1 can activate multiple signaling pathways and promote epithelial cell metastasis by inducing the EMT in CRC (21). This suggests that CTHRC1 may be a potential therapeutic target for CRC.

\section{Materials and methods}

Cells and reagents. CRC cell lines DLD-1 and LoVo, and the human intestinal epithelial cell line NCM460, were obtained from the Fuheng Cell Center (Shanghai, China). NCM460 and DLD-1 cells were cultured in Dulbecco's modified Eagle's medium (Thermo Fisher Scientific, Inc.). LoVo cells were cultured in RPMI-1640 medium (Gibco; Thermo Fisher Scientific, Inc.). All media contained $10 \%$ fetal bovine serum (FBS; Gibco; Thermo Fisher Scientific, Inc.), 100 U/ml penicillin and $100 \mathrm{mg} / \mathrm{ml}$ streptomycin. All cells were cultured in a standard humidified incubator at $37^{\circ} \mathrm{C}$ with a $5 \% \mathrm{CO}_{2}$ atmosphere. Aladdin Industrial Corporation supplied the CVB-D (cat. no. C117989). CVB-D was dissolved in methanol to produce a $71 \mathrm{mmol} / 1$ stock solution.

MTT assay. CVB-D cytotoxicity was determined in CRC and NCM460 cells via MTT assay. DLD-1, LoVo and NCM460 cells were seeded into 96 -well plates $\left(5 \times 10^{3}\right.$ per well) and cultured at $37^{\circ} \mathrm{C}$ for $24 \mathrm{~h}$ until the cells adhered to the plates. Different doses of CVB-D (0, 10, 15, 20, 25, 30, 35, 40, 45 and
$50 \mu \mathrm{M})$ were added to the plates and cultured at $37^{\circ} \mathrm{C}$ for 24 or $48 \mathrm{~h}$. MTT solution ( $5 \mathrm{mg} / \mathrm{ml} ; 20 \mu \mathrm{l} /$ well; Sigma-Aldrich, Merck $\mathrm{KGaA}$ ) was then added and the plates were incubated at $37^{\circ} \mathrm{C}$ for $4 \mathrm{~h}$. DMSO (150 $\mu \mathrm{l} /$ well $)$ was subsequently added to dissolve the formazan crystals. Absorbance at a wavelength of $490 \mathrm{~nm}$ was detected using a Microporous Plate automatic regulator (ELx808; BioTek Instruments). The formula used for calculating the cell proliferation inhibition rate was as follows: Inhibition rate $(\%)=[1-\mathrm{A} 490$ (test)/A490 (blank)] x100\%. Three biologically independent experiments were conducted.

Plate colony-forming assay. CRC cells were seeded in a 6-cm diameter dish (1,000 cells/dish) and incubated for $24 \mathrm{~h}$. CRC cells were then incubated with different doses of CVB-D $(0,20$, 30 and $40 \mu \mathrm{M})$ at $37^{\circ} \mathrm{C}$ for $5 \mathrm{~h}$. The CVB-D was then removed and replaced with normal culture medium. After 14 days, the cells were fixed in methanol at room temperature for $15 \mathrm{~min}$ and stained with $0.5 \%$ crystal violet at room temperature for $10 \mathrm{~min}$. Three biologically independent experiments were performed.

Cell cycle assay. Adherent CRC cells were treated with CVB-D $(30 \mu \mathrm{M})$ at $37^{\circ} \mathrm{C}$ for $48 \mathrm{~h}$, then harvested and fixed in $75 \%$ ethanol at $4^{\circ} \mathrm{C}$ overnight. The cells were digested and stained using RNase and propidium iodide (4A Biotech), respectively. The cells were stained at $37^{\circ} \mathrm{C}$ for $30 \mathrm{~min}$ in the dark. The cell cycle was analyzed using a flow cytometer. ModFit LT V5.0 software (Verity Software House, Inc.) was used for analysis. Three biologically independent experiments were conducted.

Apoptosis assay. CRC cells were attached to a six-well plate $\left(2.5 \times 10^{5}\right.$ cells/well $)$ and incubated overnight. Cells were then treated with different doses of CVB-D $(0,20,30$ and $40 \mu \mathrm{M})$ at $37^{\circ} \mathrm{C}$ for $48 \mathrm{~h}$, and then harvested and stained using an Annexin V-FITC/PI Apoptosis Detection kit (cat. no. FXP018; $4 \mathrm{~A}$ Biotech) according to the manufacturer's protocol. A flow cytometer was used to analyze apoptosis followed by analysis with FACS DiVa 6.1.3 software (BD Biosciences). Three biologically independent experiments were conducted.

Wound healing assay. The 90\% confluent monolayer of serum-starved CRC cells was scraped with a $200-\mu 1$ pipette tip and then treated with CVB-D $(0,10$ and $15 \mu \mathrm{M})$ at $37^{\circ} \mathrm{C}$ for $24 \mathrm{~h}$. Subsequently, photographs of the wound widths were obtained at different time periods $(0,24$ and $48 \mathrm{~h})$ using a light microscope (magnification, $\mathrm{x} 100$ ). The migration distances were analyzed by ImageJ V1.8.0 software (National Institutes of Health). Three biologically independent experiments were conducted.

Transwell assay. Cell migration and invasion experiments were conducted using Transwell chambers (BD Biosciences). CRC cells were cultured in six-well plates with different doses of CVB-D $(0,10$ and $15 \mu \mathrm{M})$ at $37^{\circ} \mathrm{C}$ for $24 \mathrm{~h}$. Culture medium $(500 \mu \mathrm{l})$ containing $10 \% \mathrm{FBS}$ was added to the lower chamber. Pretreated CRC cells were resuspended in serum-free medium (10,000 cells/200 $\mu \mathrm{l}$ medium) and injected into the upper chamber. Unlike migration analysis, $50 \mu$ l Matrigel (BD Biosciences) was used to cover the upper side of the polycarbonate membrane for the invasion assay. 
Following incubation for $48 \mathrm{~h}$, migrated or invaded cells were fixed in methanol at room temperature for $15 \mathrm{~min}$. Cells on the polycarbonate membrane were wiped off, and cells under the polycarbonate membrane were stained with $0.5 \%$ crystal violet solution at room temperature for $20 \mathrm{~min}$. Under the light microscope, five fields (magnification, x100) were randomly selected, and the mean number of cells was calculated and used for statistical analysis. Three biologically independent experiments were conducted.

Western blot analysis. CRC cells were treated with different concentrations of CVB-D $(0,20,30$ and $40 \mu \mathrm{M})$ at $37^{\circ} \mathrm{C}$ for $48 \mathrm{~h}$, and then lysed with RIPA buffer (cat. no. P0013B; Beyotime Institute of Biotechnology) containing protease and phosphatase inhibitors. Following $10 \mathrm{~min}$ of centrifugation at $12,000 \mathrm{x} \mathrm{g}$ and $4^{\circ} \mathrm{C}$, the supernatants were transferred to new test tubes. A Bradford Protein assay kit (cat. no. P0012S) was used to measure the protein concentration. Proteins (25 $\mu \mathrm{g} /$ lane) were separated by $10 \%$ SDS-PAGE (Beyotime Institute of Biotechnology) and then eletrotransferred onto polyvinylidene difluoride membranes (EMD Millipore). Membranes were blocked with 5\% non-fat milk/TBS-Tween-20 for $1 \mathrm{~h}$ at room temperature and then incubated with the corresponding primary antibodies overnight at $4^{\circ} \mathrm{C}$. The antibodies used were as follows: CTHRC1 rabbit polyclonal antibody (1:1,000; cat. no. 16534-1-AP; Proteintech Group Inc.), phosphorylated (p)-AKT (Ser473) rabbit polyclonal antibody (1:1,000; cat. no. 4060S; Cell Signaling Technology, Inc.), p-ERK (Thr202/Tyr204) rabbit polyclonal antibody $(1: 1,000$; cat. no. 4370T; Cell Signaling Technology, Inc.), E-cadherin rabbit polyclonal antibody (1:1,000; cat. no. 20874-1-AP; Proteintech Group Inc.), N-cadherin rabbit polyclonal antibody (1:1,000; cat. no. 22018-1-AP; Proteintech Group Inc.), vimentin rabbit polyclonal antibody $(1: 1,000$; cat. no. 10366-1-AP; Proteintech Group Inc.), matrix metalloproteinase (MMP)2 rabbit polyclonal antibody $(1: 1,000$; cat. no. 10373-2-AP; Proteintech Group Inc.), MMP9 rabbit polyclonal antibody (1:1,000; cat. no. 10375-2-AP; Proteintech Group Inc.), CD133 rabbit polyclonal antibody (1:1,000; cat. no. 66666-1-Ig; Proteintech Group Inc.), cyclin D1 rabbit polyclonal antibody (1:1,000; cat. no. 60186-1-Ig; Proteintech Group Inc.), B-cell lymphoma 2 (Bcl-2)-associated X protein (BAX) rabbit polyclonal antibody (1:1,000; cat. no. 50599-2-Ig; Proteintech Group Inc.), Bcl-2 rabbit polyclonal antibody (1:1,000; cat. no. 12789-1-AP; Proteintech Group Inc.), ZEB1 rabbit polyclonal antibody (1:1,000; cat. no. 21544-1-AP; Proteintech Group Inc.), ZEB2 rabbit polyclonal antibody (1:1,000; cat. no. 14026-1-AP; Proteintech Group Inc.), Snail rabbit polyclonal antibody (1:1,000; cat. no. 13099-1-AP; Proteintech Group Inc.), Slug rabbit polyclonal antibody (1:1,000; cat. no. 12129-1-AP; Proteintech Group Inc.), AKT rabbit polyclonal antibody (1:1,000; cat. no. 10176-2-AP; Proteintech Group Inc.), ERK rabbit polyclonal antibody (1:1,000; cat. no. 16443-1-AP; Proteintech Group Inc.), and $\beta$-actin mouse polyclonal antibody $(1: 1,000$; cat. no. TA-09; OriGene Technologies, Inc.). The membranes were then incubated with peroxidase-conjugated goat anti-rabbit secondary antibody (1:10,000; cat. no. ZB-2301; ZSGB-BIO) or peroxidase-conjugated goat anti-mouse secondary antibody (1:10,000; cat. no. ZB-2305; ZSGB-BIO) for $1 \mathrm{~h}$ at room temperature. Protein signals were visualized using enhanced chemiluminescence substrate (Thermo Fisher Scientific, Inc.) with a Bio-Rad gel imaging system (Bio-Rad Laboratories, Inc.). Image Lab version 2.0.1 (Bio-Rad Laboratories, Inc.) was used for quantification of the western blot data. Three biologically independent experiments were conducted.

RNA sequencing (RNA-seq) experiments. DLD-1 cells were inoculated into a $6-\mathrm{cm}$ culture dish during the exponential growth phase $\left(2.5 \times 10^{5}\right.$ cells/well $)$ and cultured overnight. After $48 \mathrm{~h}$ at $37^{\circ} \mathrm{C}$ of CVB-D $(20 \mu \mathrm{M})$ treatment, a cell suspension with $<25 \%$ of the total cells was selected as the experimental group. The cells were digested with $1.5 \mathrm{ml}$ TRIzol reagent (Invitrogen; Thermo Fisher Scientific, Inc.), then collected and transferred to $-80^{\circ} \mathrm{C}$ for storage. This sample collection step was repeated three times. The next steps including RNA isolation, cDNA library preparation and RNA-seq were completed by KangChen Bio-tech Inc. A NanoDrop was used to measure the quantity and quality of RNA. The DNA library was constructed using an Illumina kit, based on the KAPA stranded RNA-Seq Library, and enrichment of RNA was performed with oligo(dT) magnetic beads. The first-strand cDNA was generated by random primer inversion, and dUTP was added for second-strand cDNA synthesis. The final library was obtained by PCR amplification. Agilent 2100 was used to detect the quality of the constructed library, and quantitative PCR was used to quantify the library. After a series of strict quality control steps, the trimmed data were compared to the reference genome.

Bioinformatics prediction. GEPIA2 (http://gepia2.cancer-pku. cn/\#index) analysis included analyses of the differential expression of tumors/normal tissues, the survival of patients and multiple gene comparisons. The human protein atlas (http://www.proteinatlas.org) was employed to evaluate CTHRC1 protein expression in COAD tissues. The Venny online tool (https://bioinfogp.cnb.csic.es/tools/venny/) was used to identify common data in different groups of data. Gene Ontology (GO) (http://www.geneontology.org) and Kyoto Encyclopedia of Genes and Genomes (KEGG) pathway (http://www.genome.jp/kegg) enrichment analyses were performed based on the downregulated differentially expressed genes (DEGs) obtained from RNA-seq experiments. The GO terms covers three domains: Biological Process (BP), Cellular Component (CC) and Molecular Function (MF). The targets of CVB-D should comply with the following requirements: i) They were oncogenes; ii) they were overexpressed in COAD; and iii) the overexpressed oncogenes were accompanied by a decrease of OS and/or DFS.

Tumor xenograft experiments. A total of six female BALB/c nude mice (5-6-weeks old; weight, $18 \mathrm{~g}$ ) from Vital River Laboratory Animal Technology Co., Ltd. were selected and reared in a pathogen-free environment. Mice were housed at room temperature in a 12-h light/dark cycle and had access to food and water ad libitum. The Medical Ethics Committee of Harbin Medical University (Harbin, China) approved the animal protocol. Following intraperitoneal injection of $1 \%$ pentobarbital sodium into mice $(50 \mathrm{mg} / \mathrm{kg}$ ) (28), DLD-1 cells $\left(0.1 \mathrm{ml} ; 1 \times 10^{7}\right.$ cells $\left./ \mathrm{ml}\right)$ were resuspended in culture medium 
A
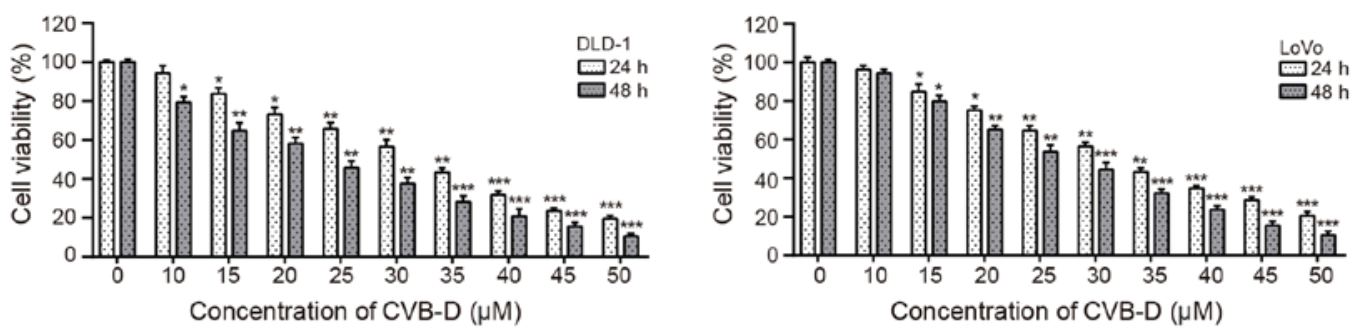

$\mathrm{B}$

DLD-1
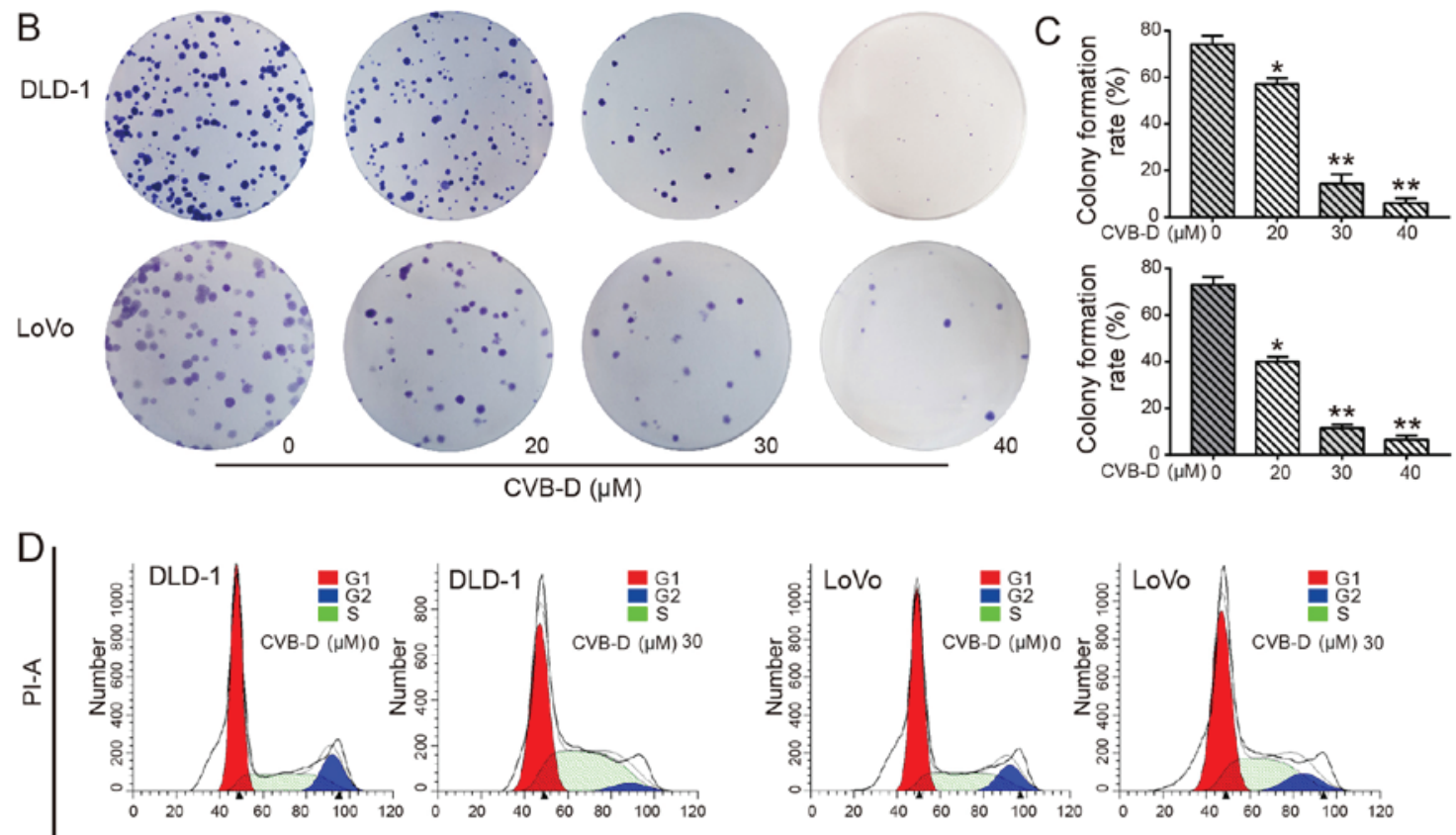

FITC-A
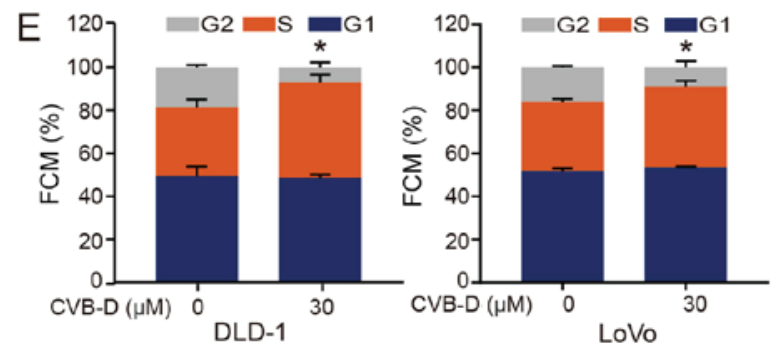

F
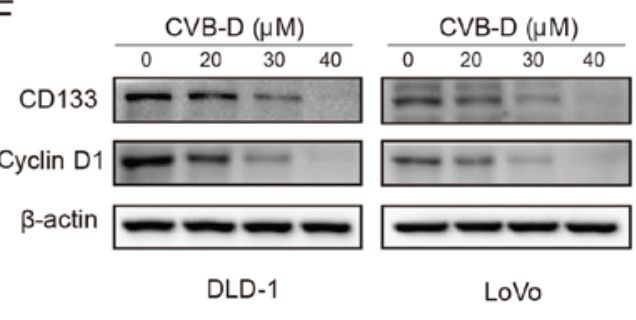

Figure 1. Effect of CVB-D on the viability of CRC cells. (A) CRC cells were treated with 0-50 $\mu \mathrm{M} \mathrm{CVB-D}$ for 24 or $48 \mathrm{~h}$, and the viability of CRC cells was determined by MTT assay. (B) Effect of treatment with 0-50 $\mu \mathrm{M}$ CVB-D for $5 \mathrm{~h}$ on colony formation of CRC cells and (C) the corresponding statistical analysis. (D) Cell cycle analysis of CRC cells treated with CVB-D (30 $\mu \mathrm{M}, 48 \mathrm{~h})$ was performed by flow cytometry and (E) the results were statistically analyzed. (F) Western blot analysis of the expression of CD133 and cyclinD1 in CRC cells treated with different concentrations of CVB-D. $\beta$-actin was used as the loading control. Data are presented as mean \pm standard deviation $(n=3)$. $P<0.05,{ }^{* *} \mathrm{P}<0.01,{ }^{* * * *} \mathrm{P}<0.001$ vs. untreated control group. CVB-D, cyclovirobuxine $\mathrm{D}$; CRC, colorectal cancer; PI, propidium iodide; FMC, flow cytometry.

and subcutaneously implanted into the flanks of mice in equal volumes to establish tumor xenografts. Mice were randomly divided into treatment and control groups (three mice in each group) 7 days after xenograft establishment. The experimental group and the negative control group were intraperitoneally injected with $15 \mathrm{mg} / \mathrm{kg}$ CVB-D and PBS, respectively, once a day for 4 weeks. Evaluation of tumor growth, angiogenesis, microangiogenesis and tumor hardness was performed using B-ultrasound, color Doppler flow imaging (CDFI), color power angiography (CPA), and ultrasonic elastosonography (USE). Finally, mice were sacrificed and the tumors, hearts, livers, spleens, lungs were collected for pathological examination at the end of the experiment.
Immunohistochemistry (IHC), hematoxylin and eosin (H\&E) staining and TUNEL apoptosis detection. For IHC, briefly, formaldehyde-fixed (at room temperature for $48 \mathrm{~h}$ ) and paraffin-embedded tumour tissues were sliced into $5-\mu \mathrm{m}$-thick sections. The sections were deparaffinized by xylene, rehydrated in a graded series of ethanol $(100,95,85$ and $75 \%$ ), incubated with $3 \% \mathrm{H}_{2} \mathrm{O}_{2}$ for $30 \mathrm{~min}$, and blocked with 3\% bovine serum albumin at room temperature for $1 \mathrm{~h}$. Sections were then immunostained with anti-Ki-67 antibody (polyclonal, rabbit anti-human IgG; 1:200; cat. no. WL01384; Wanleibio Co., Ltd.), CTHRC1 rabbit polyclonal antibody (1:200; cat. no. 16534-1-AP; Proteintech Group Inc.), Bcl-2 rabbit polyclonal antibody (1:100; cat. no. WL01556; Wanleibio 

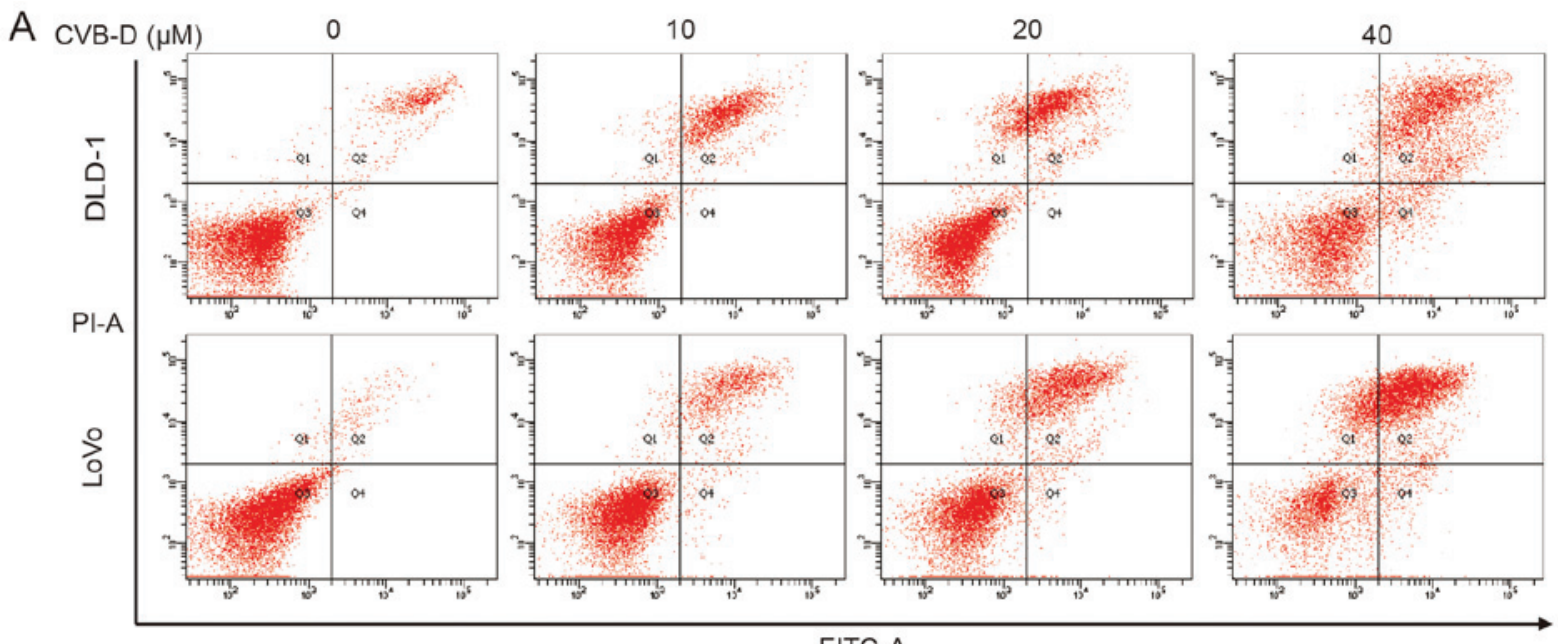

FITC-A

B

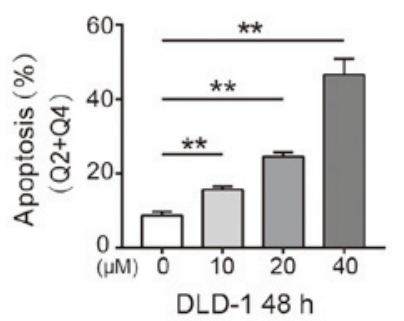

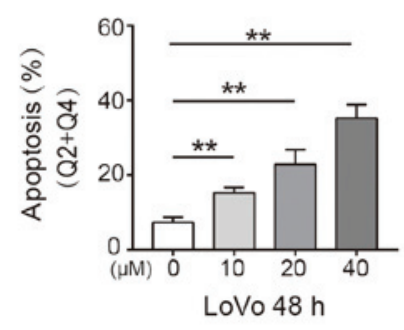

Figure 2. Effect of CVB-D on the apoptosis of CRC cells. (A) The apoptosis of CRC cells treated with CVB-D (0-40 $\mu$ M, 48 h) was determined by Annexin V-FITC/PI double staining assay. (B) The percentage of apoptotic cells was determined. (C) The expression levels of Bcl-2 and Bax in CRC cells treated with CVB-D $(0-40 \mu \mathrm{M}, 48 \mathrm{~h})$ were detected by western blot assay. $\beta$-actin was used as the loading control. ${ }^{* *} \mathrm{P}<0.01$. CVB-D, cyclovirobuxine $\mathrm{D}$; CRC, colorectal cancer; PI, propidium iodide.

Co., Ltd.), N-cadherin rabbit polyclonal antibody (1:100; cat. no. WL01047; Wanleibio Co., Ltd.), cyclin D1 rabbit polyclonal antibody (1:200; cat. no. WL01435; Wanleibio Co., Ltd.) and HIF-1 $\alpha$ rabbit polyclonal antibody (1:500; cat. no. WL01607; Wanleibio Co., Ltd.) at $4^{\circ} \mathrm{C}$ overnight. The colour was developed with a 3,3'-diaminobenzidine Substrate kit (cat. no. DA1010; Beijing Solarbio Science and Technology Co., Ltd.), and the sections were counterstained with hematoxylin at room temperature for $3 \mathrm{~min}$. Five visual fields on each slide were randomly selected and photographed under a light microscope (magnification, x400).

For H\&E, briefly, tissues were fixed at room temperature for $48 \mathrm{~h}$ and embedded in paraffin, sliced into 5 - $\mu \mathrm{m}$-thick sections, stained with hematoxylin at room temperature for 5 min, treated with $1 \%$ acid ethanol for $3 \mathrm{sec}$, washed with distilled water, dyed with eosin solution at room temperature for 3 min, dehydrated with graded alcohol and cleaned with xylene. After drying, five visual fields were randomly selected on each slide and photographed using a light microscope (magnification, x200).

Apoptosis in pathological tissues was detected using a TUNEL Apoptosis Detection kit (Wanlei Co., Ltd.; www. wanleibio.cn), according to the manufacturer's protocol. Five visual fields were randomly selected on each slide and photographed using a light microscope (magnification, $\mathrm{x} 400)$.

Transient transfection of small interfering RNA (siRNA). CTHRC1 expression was silenced by siRNA. CTHRC1 siRNA and negative control siRNA was synthesized by General Biosystems, Inc. and $100 \mathrm{nM}$ was transfected into DLD-1 and LoVo cells using Lipofectamine ${ }^{\circledR} 2000$ (Invitrogen; Thermo Fisher Scientific, Inc.). The cells were incubated for $48 \mathrm{~h}$ and then used in subsequent experiments. The siRNA sequences are presented in Table SI.

Statistical analysis. Statistical analysis was conducted using SPSS 22.0 software (IBM Corp.). Data are presented as the mean \pm standard deviation. The difference of means between two groups was assessed using Student's t-test. The difference of means between multiple groups was assessed using one-way analysis of variance, and a comparison between two groups was assessed using Bonferroni's correction. $\mathrm{P}<0.05$ was considered a statistically significant difference.

\section{Results}

$C V B-D$ suppresses $C R C$ cell proliferation. To evaluate the inhibitory effects of CVB-D on cell viability, CRC cells were exposed to increasing concentrations $(0-50 \mu \mathrm{M})$ of CVB-D for 24 and $48 \mathrm{~h}$. The results demonstrated that CVB-D significantly inhibited the CRC cell viability in a dose-dependent manner (Fig. 1A). According to probit regression analysis, the $\mathrm{IC}_{50}$ of CVB-D in DLD-1 and LoVo cells at $48 \mathrm{~h}$ was $\sim 23.20$ and $\sim 26.12 \mu \mathrm{M}$, respectively. Significant dose-dependent reductions in colony formation rate were also observed in DLD-1 and LoVo cells treated with CVB-D, as determined by colony formation assay (Fig. 1B), possibly due to a decrease 
A

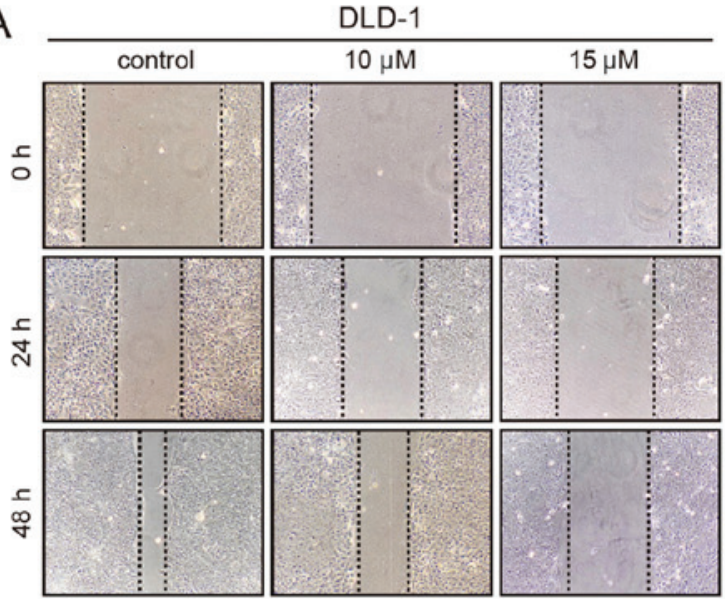

B

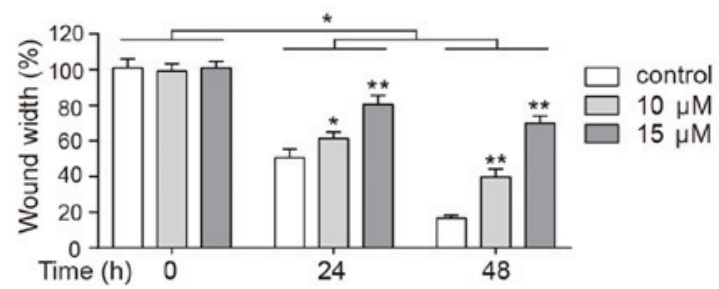

$\mathrm{C}$

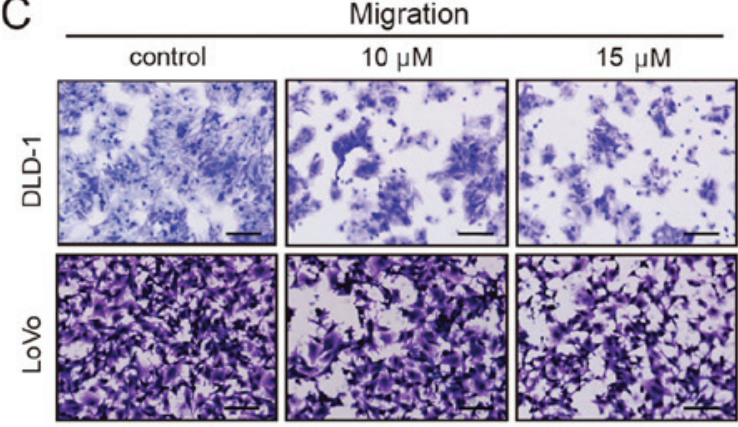

D

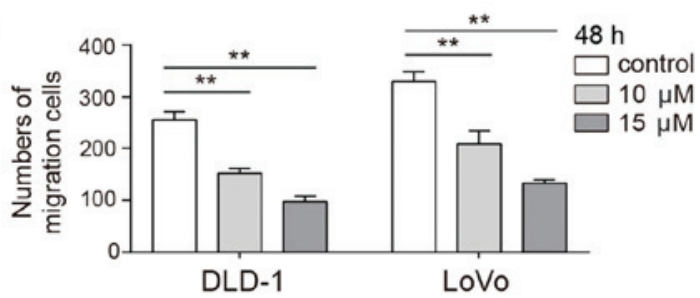

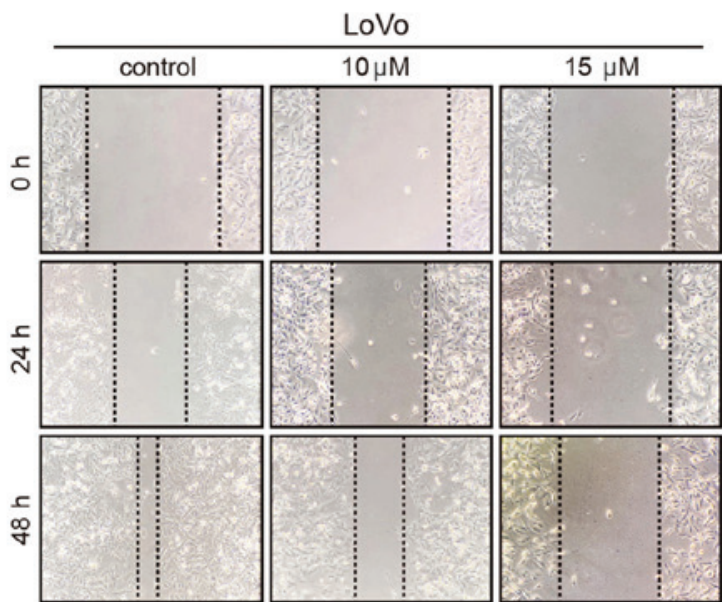

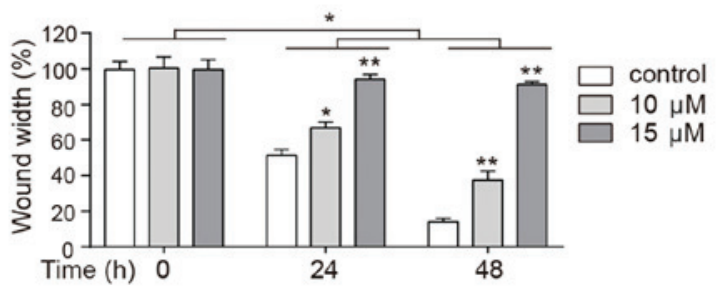

Invasion
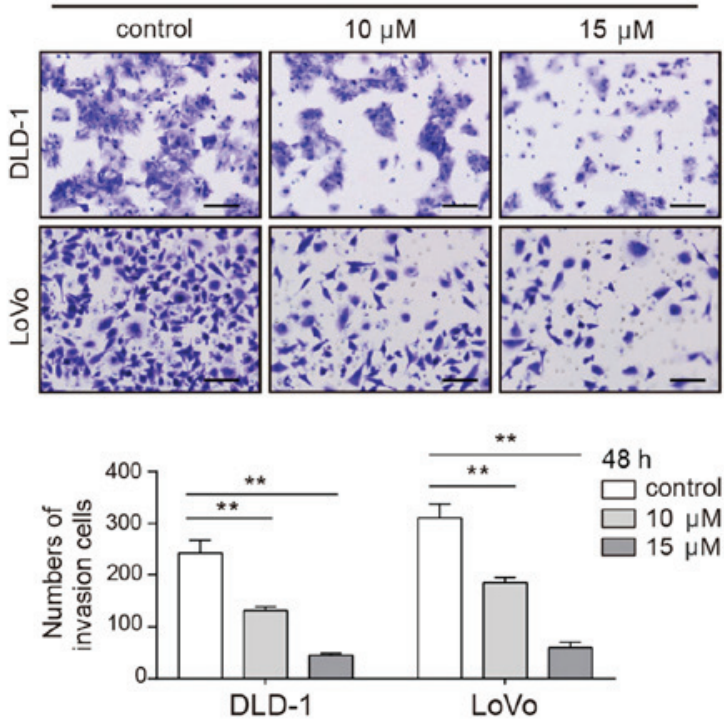

Figure 3. Effect of CVB-D on the mobility of CRC cells. (A) Wound healing assay of CRC cells following treatment with CVB-D at non-lethal concentrations $(0-15 \mu \mathrm{M}, 24 \mathrm{~h})$, and (B) the quantification of the wound widths. Magnification, $\mathrm{x} 100$. (C) Migration and invasion assays of CRC cells following pre-treatment with CVB-D at non-lethal concentrations $(0-15 \mu \mathrm{M})$ for $24 \mathrm{~h}$. Magnification, $\mathrm{x} 100$. Scale bar, $100 \mu \mathrm{m}$. (D) Results of the migration and invasion assays were statistically analyzed. Data are represented as mean \pm standard deviation $(\mathrm{n}=3)$. ${ }^{*} \mathrm{P}<0.05,{ }^{* *} \mathrm{P}<0.01$ vs. control group. CVB-D, cyclovirobuxine $\mathrm{D}$; CRC, colorectal cancer.

in stemness. CD133 is a transmembrane glycoprotein that is considered to be a surface marker of tumor stem cells and is overexpressed in CRC (29). Patients with CRC with high CD133 expression are prone to metastasis and recurrence $(30,31)$. The present results demonstrated that CD133 expression significantly decreased with an increase in drug concentration (Figs. 1F and S2), indicating that stemness may be suppressed by CVB-D. In addition, CVB-D had an insignificant effect on the viability of NCM460 cells (Fig. S1).

In cancer, control of the cell cycle is altered, leading to uncontrolled cell proliferation, thereby making the cell cycle an important target of cancer treatment (32). The overexpression of cyclin D1 in patients with CRC indicates a poor prognosis (33). Therefore, enhancing the expression of cyclin D1 and/or inhibiting its degradation is another important mechanism by which oncogenic signaling pathways, including the PI3K/AKT signaling pathway, regulate the proliferation and growth of tumor cells $(34,35)$. The regulation of cyclin D1 expression is a dynamic process in the cell. The expression levels of cyclin D1 in the G1-S-G2 phases are high, low, and low, respectively. The expression of cyclin $\mathrm{D} 1$ is low in the S phase; however, it is not the decrease of cyclin D1 expression that leads to the stagnation of $S$ phase cells (36). The cell cycle experiments demonstrated that CRC 


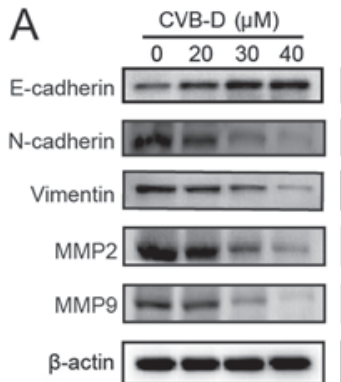

DLD-1

C

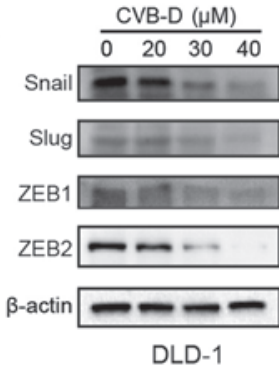

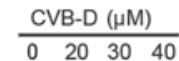

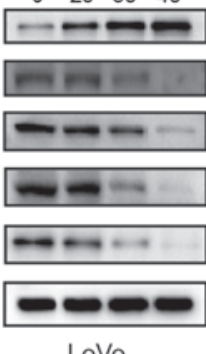

LoVo

B
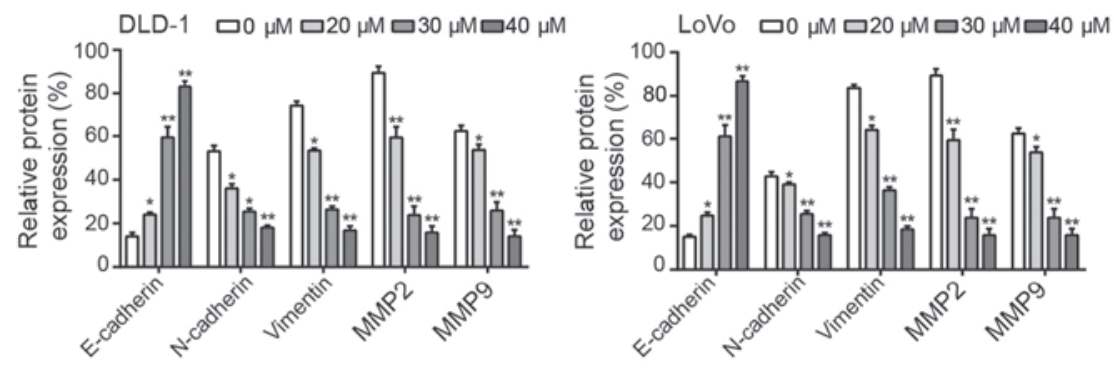

D
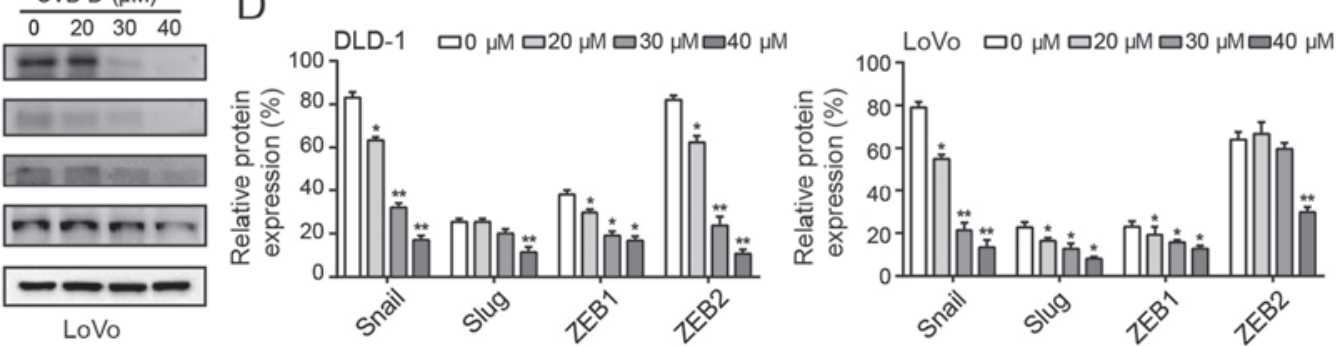

Figure 4. Inhibition of EMT-associated proteins by CVB-D. (A and B) The expression levels of EMT-related molecular markers were detected following CVB-D $(0-40 \mu \mathrm{M}, 48 \mathrm{~h})$ treatment. (C and D) The expression levels of EMT-related transcription factors were detected following CVB-D treatment $(0-40 \mu \mathrm{M}$, $48 \mathrm{~h}$ ). Data are represented as mean \pm standard deviation $(\mathrm{n}=3) .{ }^{*} \mathrm{P}<0.05,{ }^{* *} \mathrm{P}<0.01$ vs. control group. CVB-D, cyclovirobuxine $\mathrm{D}$; EMT, epithelial-mesenchymal transition; MMP, matrix metalloproteinase; ZEB, zinc finger E-box-binding homeobox.

cells were arrested at the S phase following CVB-D treatment, with a significantly higher percentage of cells in this phase (Fig. 1D and E). The expression of cyclin D1 in CRC cells was decreased following CVB-D treatment, as shown by western blot analysis (Figs. 1F and S2).

CVB-D induces apoptosis of CRC cells. Apoptosis is the key steady-state mechanism that balances cell division and cell death to restrain the uncontrolled proliferation of cancer cells; thus, induction of apoptosis is an important mechanism of anticancer drugs (37). The present study found that CVB-D significantly induced apoptosis of CRC cells in a dose-dependent manner (Fig. 2A and B). Western blot analysis demonstrated that $\mathrm{Bcl}-2$ expression significantly decreased and Bax expression significantly increased in CRC cells following treatment, indicating that CVB-D induced apoptosis (Figs. 2C and S3).

$C V B-D$ decreases the mobility of CRC cells. Pro-apoptotic chemotherapeutic agents decrease the mobility of tumor cells (38). Thus, the effects of CVB-D on cell migration and invasion were evaluated by wound healing and Transwell assays. As presented in Fig. 3A and B, the wound in the untreated cells healed rapidly, while the wound of the CVB-D-treated cells healed more slowly in a dose-dependent manner. Consistent with these observations, Transwell assays demonstrated a significantly dose-dependent decrease in the number of migrating and invading cells following CVB-D treatment (Fig. 3C and D). These results confirmed that CVB-D inhibited the mobility of CRC cells in a dose-dependent manner.

CVB-D affects the EMT through Snail. E-cadherin, vimentin, and $\mathrm{N}$-cadherin are main biomarkers of the EMT (39).
Western blot analysis revealed that CVB-D treatment significantly increased the expression of E-cadherin and significantly decreased the expression of vimentin and $\mathrm{N}$-cadherin (Fig. 4A and B). Degradation of the extracellular matrix (ECM) promotes the ability of malignant tumor cells to invade adjacent tissues, blood and lymphatic vessels in an MMP-dependent manner (40). In particular, MMP2 and MMP9 have been shown to promote cancer cell invasion and lymph node metastasis in gastrointestinal tumors (41). In the present study, MMP2 and MMP9 expression levels were decreased following treatment with CVB-D (Fig. 4A and B). In addition, western blotting demonstrated that CVB-D significantly decreased the expression of Snail in CRC cells in a dose-dependent manner. In DLD-1 cells, the expression of Slug was significantly inhibited when the concentration reached $40 \mu \mathrm{M}$, and the expression levels of ZEB1 and ZEB2 were reduced by CVB-D in a dose dependent manner. In LoVo cells, the expression levels of Slug and ZEB1 were decreased in a dose-dependent manner, and the expression of ZEB2 was significantly decreased at $40 \mu \mathrm{M}$. Therefore, compared with Slug, ZEB1 and ZEB2, only the expression of Snail was significantly decreased both in DLD-1 and LoVo cells in a dose-dependent manner (Fig. 4C and D). In view of these results, the present study mainly focused on Snail. The GEPIA database confirmed that Snail expression was significantly higher in COAD compared with adjacent tissue (Fig. S4A). Furthermore, a higher expression of Snail was significantly associated with a reduced OS rate of patients with COAD (Fig. S4B). These data suggested that inhibition of the EMT by CVB-D was regulated by Snail.

RNA-seq indicates that CVB-D exerts anticancer effects on CRC cells mainly by inhibiting the AKT/ERK signaling pathway. RNA-seq was used to investigate the mechanisms 

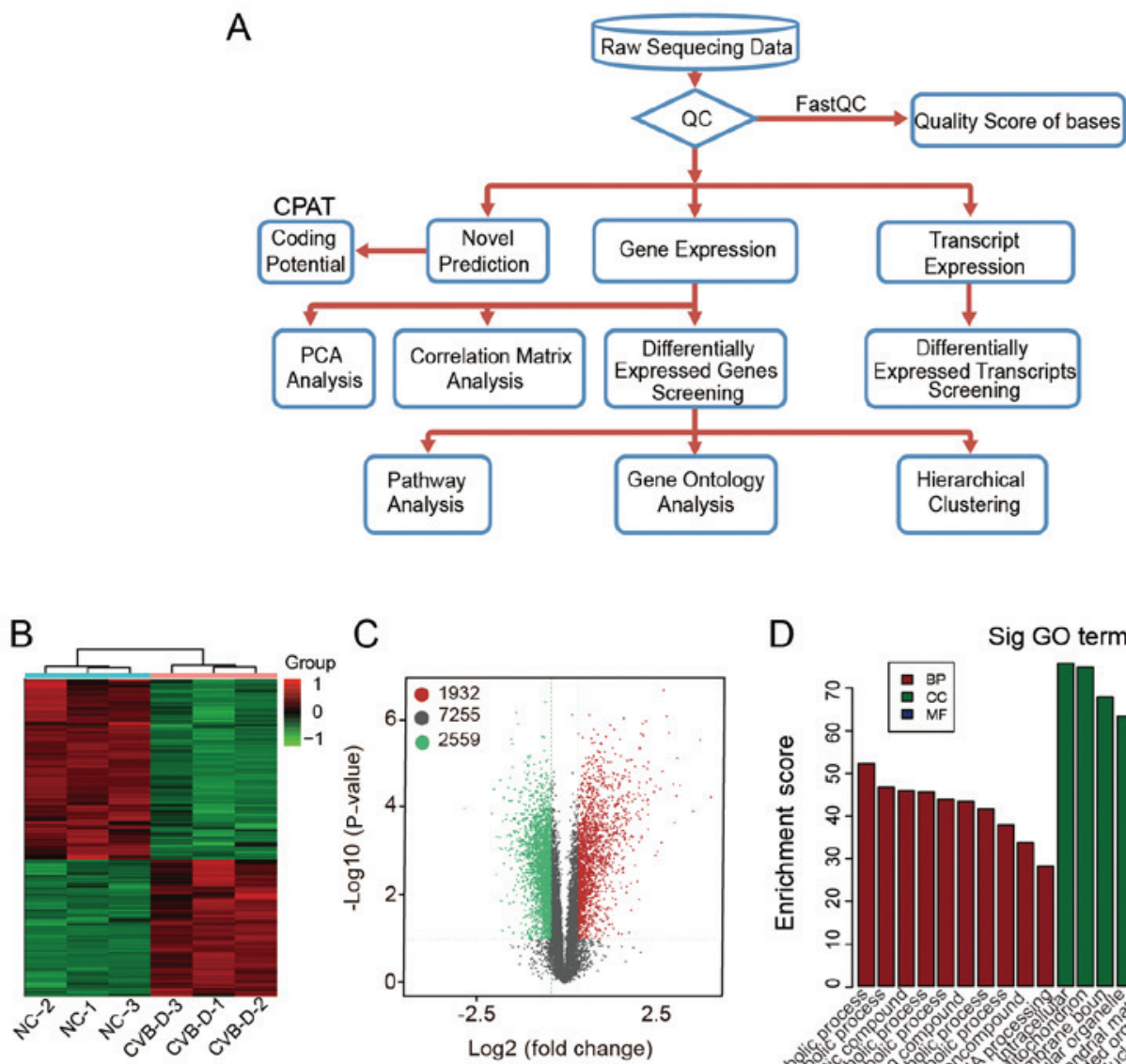

C

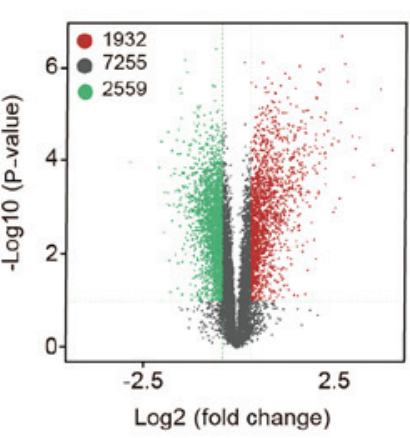

D
Sig GO terms of DEGs

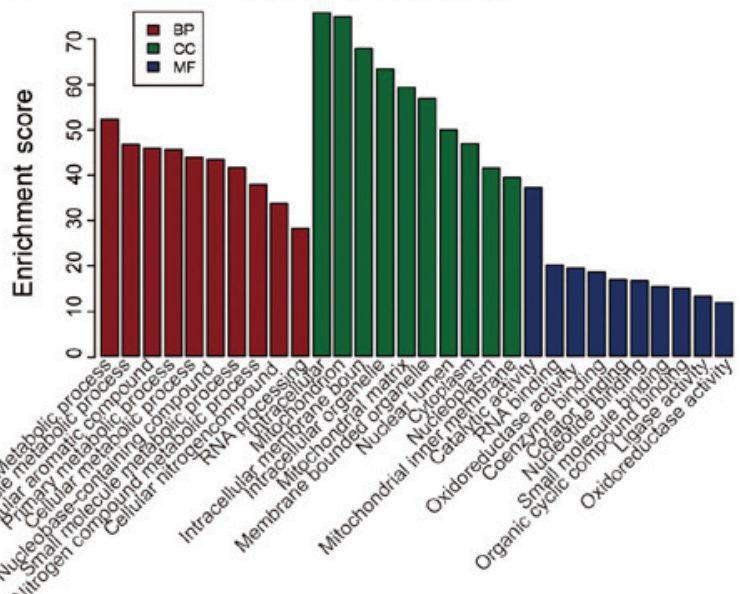

$\mathrm{E}$

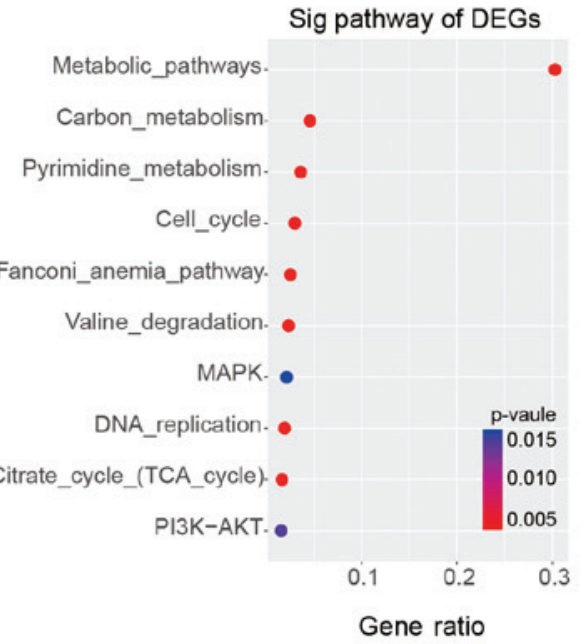

$\mathrm{F}$

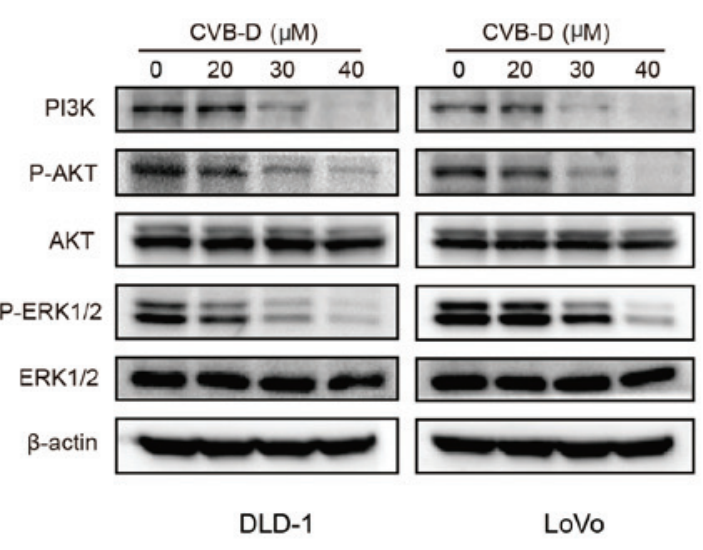

Figure 5. Analysis of RNA-seq data obtained from DLD-1 cells treated with CVB-D. (A) Experimental flowchart of RNA-seq. (B) Correlation of gene expression levels among samples. (C) A heat map shows the hierarchical clustering of DEGs in DLD-1 cells following treatment with CVB-D. Upregulated and downregulated genes are represented by red and green, respectively. (D) A volcano plot shows the number of upregulated, downregulated and non-differentially expressed genes. (E) GO analysis obtained information regarding BP, CC and MF terms associated with the downregulated DEGs (F) Western blot analysis verified the expression of major proteins of the AKT/ERK signaling pathway in CRC cells treated with CVB-D $(0-40 \mu \mathrm{M}, 48 \mathrm{~h})$. Data are presented as mean \pm standard deviation $(\mathrm{n}=3)$. BP, biological process; CC, cellular component; MF, molecular function; RNA-seq, RNA sequencing; CVB-D, cyclovirobuxine D; DEG, differentially expressed gene; GO, Gene Ontology; NC, negative control; p-, phosphorylated; sig, significant.

underlying the effects of CVB-D on CRC. The experimental flowchart is presented in Fig. 5A. Hierarchical clustering and volcano plots demonstrated that 1,932 DEGs were upregulated and 2,559 DEGs were downregulated, and 7,255 DEGs were unchanged in RNA-seq data (Fig. 5B and C). According to the functional annotation in the GO database, the downregulated DEGs were mostly enriched in the following BP terms 'metabolic process' and 'heterocycle metabolic process'; the 
A

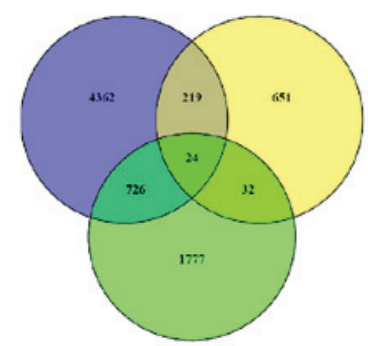

DEGs (COAD vs. normal)

Downregulated DEGs in RNA-seq

MDSG

D

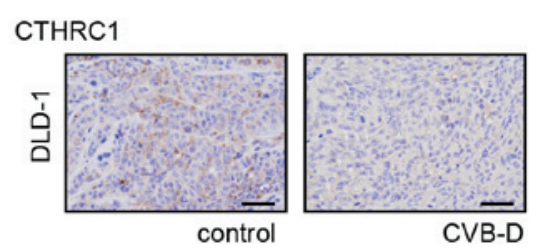

$\mathrm{F}$

DLD-1

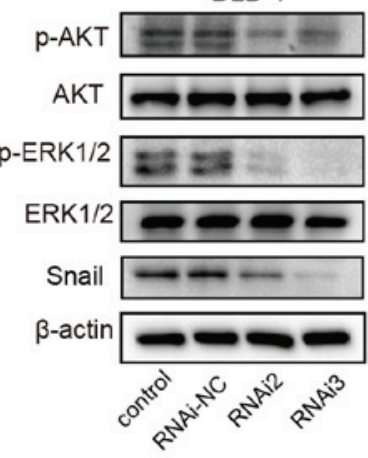

LoVo
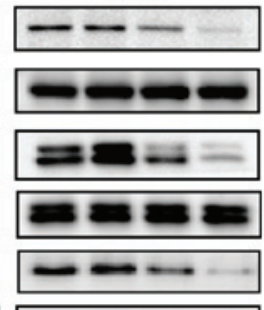

B

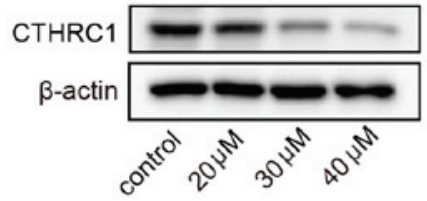

LoVo

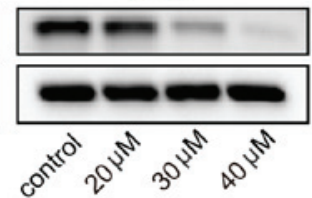

C

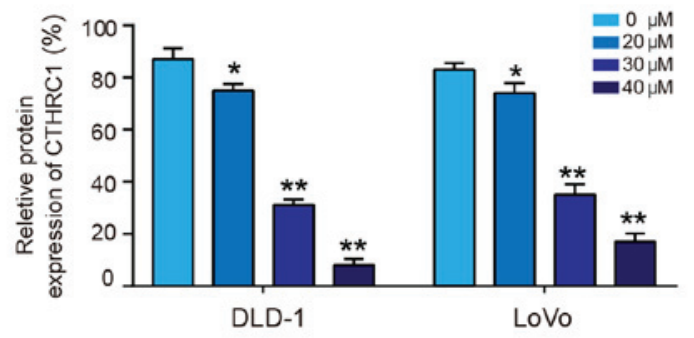

E
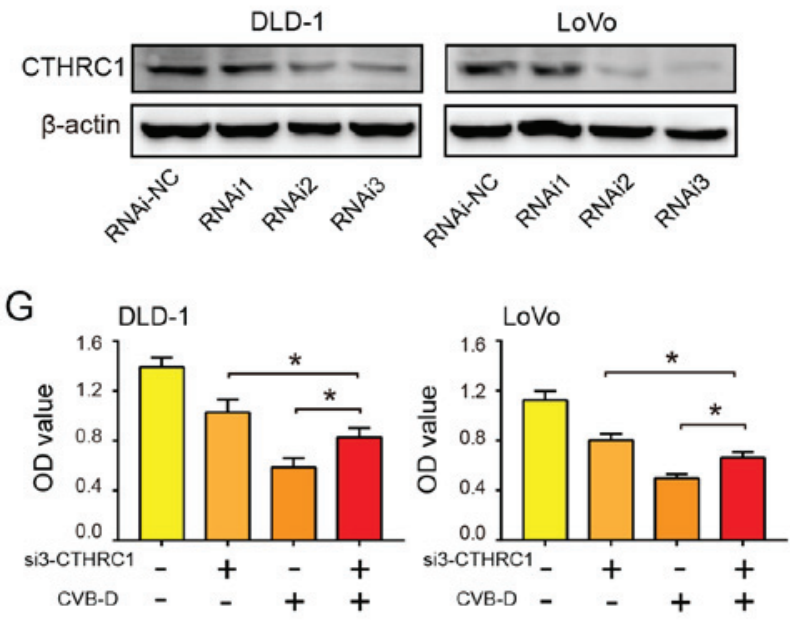

Figure 6. CTHRC1 as a therapeutic target of CRC. (A) Venn diagram showing the number of potential targets in three sets of data. (B and C) Western blot analysis was performed to evaluate the expression of CTHRC1 in CRC cells following treatment with CVB-D (0-40 $\mu \mathrm{M})$ for $48 \mathrm{~h}$. (D) Immunohistochemical analysis of CTHRC1 expression after treatment with CVB-D (15 mg/kg, once a day for a total of 4 weeks) in the DLD-1 xenograft tumors (magnification, x400; scale bar, $100 \mu \mathrm{M}$ ). (E) siRNA-mediated knockdown of CTHRC1 in CRC cells was evaluated by western blot assay. (F) siRNA-mediated knockdown of CTHRC1 in CRC cells resulted in reduced levels of p-AKT, p-ERK and Snail. (G) CVB-D significantly reversed the effect of CTHRC1-knockdown on the viability of CRC cell. Data are presented as mean \pm standard deviation $(n=3)$. ${ }^{*} \mathrm{P}<0.05,{ }^{* *} \mathrm{P}<0.01$ vs. untreated control group. CVB-D, cyclovirobuxine $\mathrm{D}$; DEG, differentially expressed gene; CTHRC1, collagen triple helix repeat containing 1; p-, phosphorylated; OD, optical density; si, small interfering; CRC, colorectal cancer; NC, negative control; MDSG, most differential survival genes that are associated with the overall survival and/or disease-free survival rates of patients.

CC terms 'intracellular' and 'mitochondrion'; and the MF terms 'catalytic activity' and 'RNA binding' (Fig. 5D). As cellular metabolism is the foundation of all biological activities, studying the mechanism of the metabolic process may enhance cancer treatment methods (42). The classical MAPK, PI3K-AKT and cell cycle pathway pathways were associated with the group of downregulated DEGs in the RNA-seq dataset (Fig. 5E). Western blot analysis verified that the protein expression levels of p-AKT, p-ERK1/2 and PI3K were inhibited by CVB-D in a dose-dependent manner, while, the levels of total AKT and ERK1/2 demonstrated no difference, indicating that CVB-D exerts anticancer effects on CRC by inhibiting the AKT/ERK signaling pathway (Figs. 5F and S5).

CTHRC1 is a therapeutic target of CRC cells. By mining the GEPIA2 database, a total of 5,331 DEGs related to COAD and 926 most differentially expressed survival genes associated with the OS and/or disease-free survival (DFS) rates of patients with COAD were identified. Using the Venny online tool, 24 overlapping genes were found between the two aforementioned groups of data and the downregulated genes revealed by RNA-seq (Fig. 6A). Then, potential targets were selected according to three conditions: i) They were oncogenes; ii) they were overexpressed in COAD (Fig. S6); and iii) the overexpressed oncogenes were accompanied by a decrease in OS and/or DFS (Figs. S7 and S8).

The remaining four genes, CTHRC1, C2orf70, NIFK and COMT, were considered the most likely potential targets for CVB-D. GEPIA2 was used to analyze the correlation between the four genes (CTHRC1, COMT, C2orf70 and NIFK) and transcription factors. The correlations were low between Snail and COMT ( $\left.\mathrm{r}=0.35, \mathrm{P}=1.1 \times 10^{-10}\right)$, Snail and C2orf70 $(\mathrm{r}=0.26$, $\left.\mathrm{P}=2.4 \times 10^{-6}\right)$, and Snail and NIFK $\left(\mathrm{r}=0.38, \mathrm{P}=1.6 \times 10^{-12}\right)$. However, there was a significantly high correlation between 
A

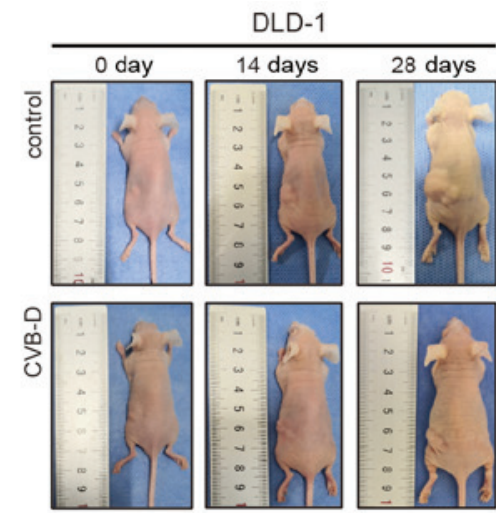

B

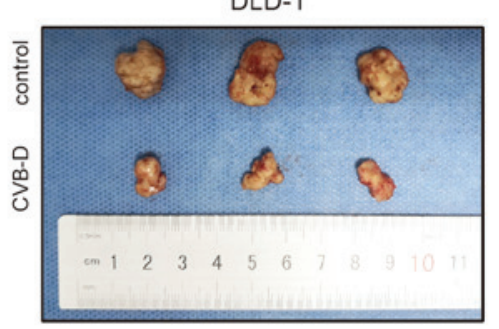

C
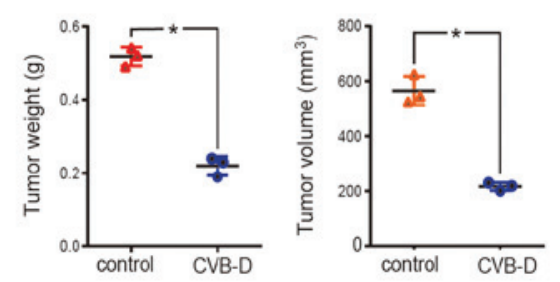

D

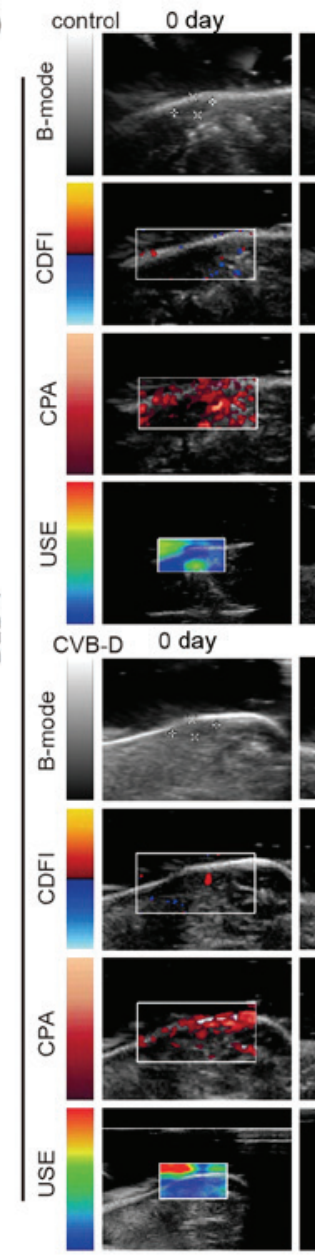

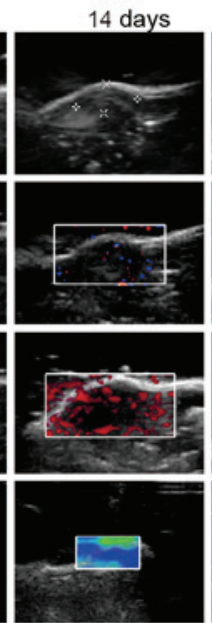

14 days
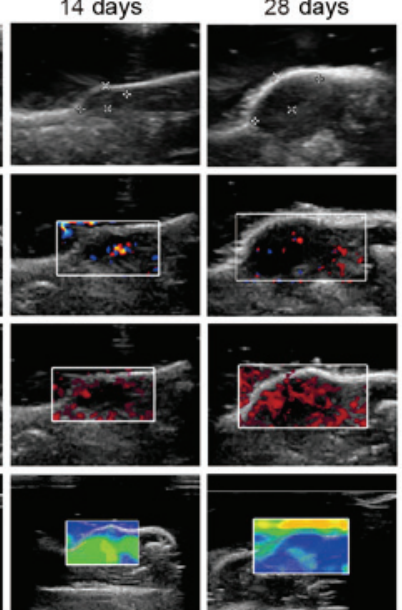

E

흘
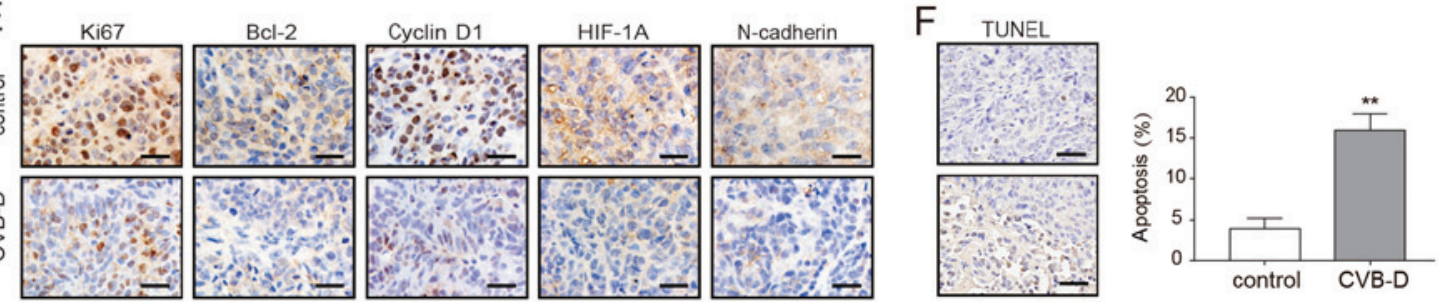

Figure 7. Inhibitory effect of CVB-D on DLD-1 xenografts in BALB/c nude mice. (A-C) The xenograft tumor model of DLD-1 cells in nude mice was established and then treated with CVB-D (15 mg/kg/day for 4 weeks). All mice were observed, and the tumor weight and volume were measured. (D) B-mode, CDFI, CPA and USE of ultrasonography were used to evaluate xenografts in the treatment and control groups. (E) The expressions of Ki67, Bcl-2, cyclin D1, HIF-1 $\alpha$ and N-cadherin were detected by immunohistochemical staining (original magnification, $x 400$; scale bar, $100 \mu \mathrm{M}$ ), (F) A TUNEL assay was used to detect apoptosis in pathological tissues. Data are presented as mean \pm standard deviation $(\mathrm{n}=3)$. ${ }^{*} \mathrm{P}<0.05$ and ${ }^{* *} \mathrm{P}<0.01$. B-mode, B-ultrasound; $\mathrm{CDFI}$, color Doppler flow imaging; CPA, color power angiography; USE, ultrasonic elastosonography; CVB-D, cyclovirobuxine D; HIF-1 $\alpha$, human inducible factor-1 $\alpha$.

Snail and CTHRC1 (r=0.69; P=3.1x10-45) (Fig. S9A). In addition, the correlation between other transcription factors and the four potential targets was also analyzed. COMT, C2orf70 and NIFK had no or low correlation with Slug, ZEB1 and ZEB2. Whereas, CTHRC1 had a certain degree of correlation with other three transcription factors (CTHRC1 and Slug $\left(\mathrm{r}=0.57, \mathrm{P}=4.5 \times 10^{-29}\right) ; \mathrm{CTHRC} 1$ and $\mathrm{ZEB} 1\left(\mathrm{r}=0.43, \mathrm{P}=1 \times 10^{-15}\right)$; CTHRC1 and ZEB2 ( $\left.\mathrm{r}=0.37, \mathrm{P}=8.7 \times 10^{-12}\right)$ (Fig. S9). The protein and mRNA expression analysis using the online databases demonstrated that the expression of CTHRC1 was significantly higher in COAD tissues compared with adjacent normal tissues (Fig. S10A and B). The CTHRC1 mRNA expression in COAD ranked 13th highest among 31 types of tumors (Fig. S10C). Patient survival analysis revealed that overexpression of CTHRC1 predicted poor OS and DFS rates in COAD (Fig. S10D). Western blotting reveled that the expression of CTHRC1 in DLD-1 and LoVo cells decreased in a dose-dependent manner following treatment with CVB-D (Fig. 6B and C). IHC results demonstrated that CTHRC1 expression in the CVB-D group was lower compared with the control group (Fig. 6D). RNAi2 and RNAi3 effectively downregulated the expression of CTHRC1 (Figs. 6E and S11). Western blot analysis demonstrated that the ratios of p-AKT/AKT and p-ERK/ERK significantly decreased after siRNA-mediated CTHRC1-knockdown; the expression level of Snail was also decreased after siRNA-mediated CTHRC1-knockdown 
A

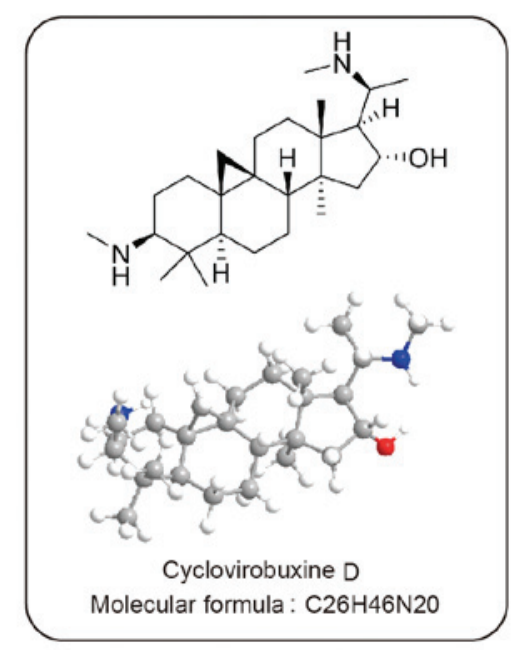

B

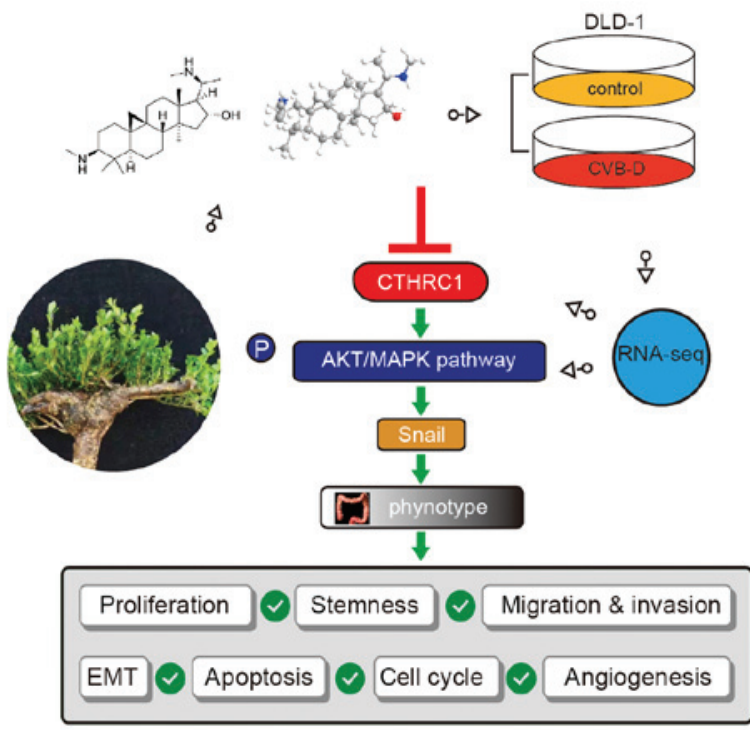

Figure 8. Chemical structure of CVB-D and its inhibitory mechanism in CRC. (A) Chemical structure and 3D structure of CVB-D. (B) The molecular mechanism of CVB-D-induced inhibition of CRC occurs via the CTHRC1-AKT/ERK-Snail signaling pathway. CVB-D, cyclovirobuxine D; CTHRC1, collagen triple helix repeat containing 1; CRC, colorectal cancer.

(Figs. 6F and S12), indicating that CTHRC1 may be located upstream of p-AKT, p-ERK and Snail. In the siRNA3 rescue experiment, siRNA3-mediated suppression of CTHRC1 in CRC cells resulted in a partial reversal of CVB-D-induced growth inhibition (Fig. 6G). These results suggest that the growth of CRC cells inhibited by CVB-D is mediated by a CTHRC1-dependent mechanism.

$C V B-D$ inhibition on the growth of CRC xenograft tumors in nude mice. The DLD-1 xenograft model was established in BALB/C nude mice to evaluate the antitumor ability of CVB-D. After 4 weeks, tumor weight and size in the CVB-D treatment group were significantly smaller compared with the control group (Fig. 7A-C). The expression levels of Ki67, cyclin D1, Bcl-2, HIF1A and N-cadherin were decreased in the CVB-D treatment compared with the control group, as detected by IHC (Fig. 7E).

In addition to dynamically observing the growth of the transplanted tumor and comparing the size of the excised tumor after execution, ultrasonic imaging (USI) was employed to dynamically detect the tumor indicators. USI can continuously and dynamically scan the lesions, and can show stereoscopic changes, which are not limited by image stratification, and does not damage the nude mice.

USI can also be evaluated systematically with multi-parameters such as Doppler and elastography (43). The ultrasound scan mode in this experiment included B-mode, CDFI, CPA and USE. The results demonstrated that tumor growth slowed after CVB-D treatment; angiogenesis decreased, especially microvascular; the elasticity score, which is highly related to tumor malignancy, decreased; and hardness of tumor decreased (Fig. 7D). The H\&E staining result revealed that the number of blood vessels in the experimental group was decreased after CVB-D treatment (Fig. S13). H\&E staining demonstrated that there were no obvious morphological changes in the heart, liver, spleen and lung (Fig. S14). The apoptosis rate of xenograft tumor cells, as detected by the
TUNEL assay, was $15.91 \%$ in the CVB-D treatment group and $3.96 \%$ in the control group, demonstrating the antitumor effects of CVB-D (Fig. 7F).

CVB-D chemical structure and the inhibitory mechanism of action in CRC. The chemical and 3D structure of CVB-D is presented in Fig. 8A. A schematic presentation of the mechanism underlying CVB-D-induced inhibition of CRC and the relevant molecular mechanism associated with the AKT/ERK-Snail signaling pathway is presented in Fig. 8B.

\section{Discussion}

It has been reported that NPCs exhibit anticancer, anti-inflammatory and anti-fibrotic effects, which inhibits pathological EMT through different cell signal transduction pathways (10). In the present study, the expression of epithelial marker E-cadherin increased following CVB-D treatment, while the mesenchymal markers $\mathrm{N}$-cadherin and vimentin decreased. Therefore, it was speculated that CVB-D can prevent EMT. In addition, the expression levels of the stemness marker CD133, invasion and metastasis related markers MMP2 and MMP9, and following CVB-D treatment as detected by western blotting. Subsequently, the expression of transcription factors, which have been demonstrated to be involved in the upstream regulation of EMT, was investigated. The consistent and significant inhibitory effect of CVB-D on Snail expression in both types of CRC cells suggests that Snail may be the optimum candidate among EMT regulatory factors. Notably, database analysis identified that the expression of Snail is higher in COAD tissues compared with paracancerous tissues, further supporting Snail as the optimum EMT regulator candidate. Additionally, high Snail expression is associated with shorter survival time and promotes the occurrence and development of CRC (44). These results suggest that CVB-D may suppress EMT by regulating the expression of Snail. 
RNA-seq techniques have been used for the study of numerous diseases, such as bladder, gastric, liver, cervical, colon and breast cancers (45-50). RNA-seq is an effective tool for sequencing transcripts using high-throughput sequencing technology. The complete mechanism of drug action may be obtained by analyzing RNA-seq data, such as the potential targets of drug action and oncogenic signaling pathways (51). Generally, antitumor agents typically inhibit oncogenic signaling pathways; therefore, the present study focused on the downregulated signaling pathways revealed by RNA-seq. According to the RNA-seq data, each pathway corresponded to a statistical P-value to indicate significance. The smaller the P-value, the more significant the association between the differential genome and the signaling pathway. The P-value of the metabolic signaling pathway was lowest in the downregulated signaling pathways; however, the purpose of this experiment was to determine the association between drugs and oncogenic signaling pathways; therefore, metabolic signaling pathways were removed. The oncogenic signaling pathways ranked high, i.e. classical MAPK and PI3K-AKT pathways, were given primary attention in the downregulated signaling pathways revealed by RNA-seq, which were demonstrated to be important signaling pathways in CRC (52). Activation of the PI3K-AKT signaling pathway regulates multiple biological processes, such as promoting invasion and metastasis of CRC (53). Abnormal activation of the RAS/Raf/MRK/ERK signaling pathway promotes the progression of colon cancer and has been identified as a novel target for tumor therapy (54). Zhao et al (55) demonstrated that CAPS1 accelerated CRC metastasis via the EMT process mediated by the PI3K/AKT/Snail signaling pathway, and also confirmed that Snail silencing can attenuate the CASP1 overexpression-induced migration and invasion of SW480 cells. Zhu et al (56) demonstrated that ZC3H13 inhibits proliferation and invasion of CRC via the Ras-ERK-Snail signaling pathway. These studies indicate that these two signaling pathways play an important role in CRC and can serve a role upstream of Snail. Western blotting confirmed that CVB-D plays an anticancer role by downregulating the AKT/ERK pathway.

It was hypothesized that drug-target genes may be differentially expressed in COAD. By mining the GEPIA2 database, a total of 5,331 DEGs in COAD and 926 MDSG were identified. Using the Venny online tool, 24 overlapping genes were found between the two aforementioned groups of data and the downregulated genes revealed by RNA-seq. Four oncogenes, including CTHRC1, C2orf70, NIFK and COMT, were further selected because they were overexpressed in COAD and associated with poor OS and/or DFS, which were considered the most likely potential targets for CVB-D. At the start of the present study, it was identified that of the EMT regulatory factors analyzed, Snail decreased most notably following CVB-D treatment; therefore, correlations among the four genes (CTHRC1, COMT, C2orf70 and NIFK) and Snail were analyzed in GEPIA2. Accordingly, a high correlation between Snail and CTHRC1 was identified. Therefore, CTHRC1 was selected as the potential therapeutic target of CVB-D for further experimental study.

CTHRC1 has been reported to be an oncogene in previous studies (21-27), and it can promote tumorigenesis by multiple mechanisms. It has been confirmed that CTHRC1 can activate the SRC and ERK signal cascades and upregulate the expression of MMP9, thus promoting the invasion of CRC cells (22). Overexpression of CTHRC1 has been found to promote the occurrence and development of cervical cancer by activating the Wnt/PCP oncogenic signaling pathway (57). CTHRC1 induces EMT changes and MMP expression through the $\mathrm{PI} 3 \mathrm{~K} / \mathrm{AKT} / \mathrm{ERK} / \mathrm{CREB}$ signaling pathway to promote the invasiveness and metastasis of hepatocellular carcinoma (58). In addition, there is a close relationship between CTHRC1 and EMT. Ni et al (21) demonstrated that CTHRC1 can promote epithelial cell metastasis by inducing the EMT in CRC cells. In a study by Kim et al (22), CTHRC1-knockdown prevented EMT and inhibited the proliferation of renal cell carcinoma. Liu et al (59) reported that CTHRC1-knockdown inhibits EMT and cell migration in glioblastoma cells. Notably, CTHRC1 can be used as a prognostic indicator for numerous tumors. $\mathrm{Gu}$ et al (60) found CTHRC1 overexpression in a large proportion of patients with gastric cancer, and the high expression was associated with gastric cancer progression and poor prognosis. Kaplan-Meier analysis by Hou et al (61) revealed that patients with epithelial ovarian cancer with high CTHRC1 expression had significantly shorter OS times. Multivariate cox analysis demonstrated that for patients with CRC, CTHRC1 could be used as an independent prognostic factor for OS (21).

In the present study, the inhibitory effect of CVB-D on the expression of CTHRC1 protein in CRC was confirmed by western blotting with cell lines and IHC of the subcutaneous transplanted tumors in nude mice. Then, CTHRC1-knockdown experiments were performed. Following siRNA-mediated knockdown, the levels of p-AKT and p-ERK were decreased, indicating that CTHRC1 is located upstream of the AKT/ERK pathway. Finally, CTHRC1-knockdown cell lines were treated with CVB-D for rescue experiments. CVB-D alone was superior to si-CTHRC1 alone in inhibiting cell viability, and the addition of CVB-D to CRC cells pretreated with CTHRC1 siRNA did not cause further inhibition. Therefore, the present experiments demonstrated that the anticancer effect of CVB-D was predominantly achieved by inhibiting the CTHRC1-AKT/ERK-Snail signal pathway.

In the past, oral or intravenous administration of traditional chemotherapy has struggled to increase local drug concentration in tumors, leading to low drug utilization and poor therapeutic effectiveness (62). The rapid development of scientific progress has resulted in the development of precise and complex nanoscale devices, which can actively drive drugs to the target at the molecular level (63). This will greatly increase the local blood concentration, further reduce toxicity and side effects, and effectively amplify multi-target anticancer mechanisms of drugs.

There were several limitations of the present study. First, due to ethical limitations, the sample size of the animal experiment was small; however, the results were consistent. Second, there were differences in CTHRC1-knockdown efficiency between the two siRNAs, which may have been due to off-target effects.

In conclusion, as an alkaloid chemotherapeutic drug, CVB-D was shown to inhibit the viability, migration, invasion and stemness of CRC cells, and induce apoptosis and cell cycle arrest. Through RNA-seq analysis, it was further confirmed 
that CVB-D can inhibit the tumorigenesis of CRC via the CTHRC1-AKT/ERK-Snail signaling pathway.

\section{Acknowledgements}

Not applicable.

\section{Funding}

This work was supported by the Scientific Planning Project of Heilongjiang Province (grant. no. 201713) and the Innovation Project of Harbin Medical University (grant. no. YJSKYCX2018-37HYD).

\section{Availability of data and materials}

The datasets used and/or analyzed during the current study are available from the corresponding author on reasonable request.

\section{Authors' contributions}

FJ, YC and DP conceived and designed the study. FJ, SR and YC performed the experiments. YZ, KS and YX performed the pathology experiments. ZL performed ultrasonic imaging experiments. FJ wrote the manuscript. All authors read and approved the final manuscript.

\section{Ethics approval and consent to participate}

All animal studies were approved by the Ethics Committee of the First Affiliated Hospital of Harbin Medical University (Heilongjiang, China) and were conducted according to the national regulations in China.

\section{Patient consent for publication}

Not applicable.

\section{Competing interests}

The authors declare that they have no competing interests.

\section{References}

1. Ferlay J, Soerjomataram I, Dikshit R, Eser S, Mathers C, Rebelo M, Parkin DM, Forman D and Bray F: Cancer incidence and mortality worldwide: Sources, methods and major patterns in GLOBOCAN 2012. Int J Cancer 136: E359-E386, 2015.

2. Liu Z, Bai Y, Xie F, Miao F and Du F: Comprehensive analysis for identifying diagnostic and prognostic biomarkers in colon adenocarcinoma. DNA Cell Biol: Feb 7, 2020 (Epub ahead of print).

3. Rasool S, Kadla SA, Rasool V and Ganai BA: A comparative overview of general risk factors associated with the incidence of colorectal cancer. Tumour Biol 34: 2469-2476, 2013.

4. Van Cutsem E, Nordlinger B and Cervantes A; ESMO Guidelines Working Group: Advanced colorectal cancer: ESMO clinical practice guidelines for treatment. Ann Oncol 21 (Suppl 5): v93-v97, 2010

5. Chen C, Xu ZQ, Zong YP, Ou BC, Shen XH, Feng H, Zheng MH, Zhao JK and Lu AG: CXCL5 induces tumor angiogenesis via enhancing the expression of FOXD1 mediated by the AKT/NF- $\kappa \mathrm{B}$ pathway in colorectal cancer. Cell Death Dis 10: 178, 2019.

6. Tomeh MA, Hadianamrei R and Zhao X: A review of curcumin and its derivatives as anticancer agents. Int J Mol Sci 20: E1033, 2019.
7. Lee SR, Jin H, Kim WT, Kim WJ, Kim SZ, Leem SH and Kim SM: Tristetraprolin activation by resveratrol inhibits the proliferation and metastasis of colorectal cancer cells. Int J Oncol 53: 1269-1278, 2018

8. Wang JY, Wang Z, Li MY, Zhang Z, Mi C, Zuo HX, Xing Y, Wu YL, Lian LH, Xu GH, et al: Dictamnine promotes apoptosis and inhibits epithelial-mesenchymal transition, migration, invasion and proliferation by downregulating the HIF-1 $\alpha$ and Slug signaling pathways. Chem Biol Interact 296: 134-144, 2018.

9. Boldbaatar A, Lee S, Han S, Jeong AL, Ka HI, Buyanravjikh S, Lee JH, Lim JS, Lee M and Yang Y: Eupatolide inhibits the TGF- $\beta 1$-induced migration of breast cancer cells via downregulation of SMAD3 phosphorylation and transcriptional repression of ALK5. Oncol Lett 14: 6031-6039, 2017.

10. Avila-Carrasco L, Majano P, Sánchez-Toméro JA, Selgas R, López-Cabrera M, Aguilera A and González Mateo G: Natural plants compounds as modulators of epithelial-to-mesenchymal transition. Front Pharmacol 10: 715, 2019.

11. Yu B, Fang TH, Lü GH, Xu HQ and Lu JF: Beneficial effect of Cyclovirobuxine D on heart failure rats following myocardial infarction. Fitoterapia 82: 868-877, 2011.

12. Lu J, Sun D, Gao S, Gao Y, Ye J and Liu P: Cyclovirobuxine D induces autophagy-associated cell death via the Akt/mTOR pathway in MCF-7 human breast cancer cells. J Pharmacol Sci 125: 74-82, 2014.

13. Wu J, Tan Z, Chen J and Dong C: Cyclovirobuxine D inhibits cell proliferation and induces mitochondria-mediated apoptosis in human gastric cancer cells. Molecules 20: 20659-20668, 2015.

14. Kalluri R and Weinberg RA: The basics of epithelial-mesenchymal transition. J Clin Invest 119: 1420-1428, 2009.

15. Savagner P: The epithelial-mesenchymal transition (EMT) phenomenon. Ann Oncol 21 (Suppl 7): vii89-vii92, 2010.

16. Kong D, Zhang F, Shao J, Wu L, Zhang X, Chen L, Lu Y and Zheng S: Curcumin inhibits cobalt chloride-induced epithelial-to-mesenchymal transition associated with interference with TGF- $\beta$ /Smad signaling in hepatocytes. Lab Invest 95: 1234-1245, 2015.

17. Li Z, Chen Y, An T, Liu P, Zhu J, Yang H, Zhang W, Dong T, Jiang J, Zhang Y, et al: Nuciferine inhibits the progression of glioblastoma by suppressing the SOX2-AKT/STAT3-Slug signaling pathway. J Exp Clin Cancer Res 38: 139, 2019.

18. Cao H, Xu E, Liu H, Wan L and Lai M: Epithelial-mesenchymal transition in colorectal cancer metastasis: A system review. Pathol Res Pract 211: 557-569, 2015.

19. Barr S, Thomson S, Buck E, Russo S, Petti F, Sujka-Kwok I, Eyzaguirre A, Rosenfeld-Franklin M, Gibson NW, Miglarese M, et al: Bypassing cellular EGF receptor dependence through epithelial-to-mesenchymal-like transitions. Clin Exp Metastasis 25: 685-693, 2008.

20. Pyagay P, Heroult M, Wang Q, Lehnert W, Belden J, Liaw L, Friesel RE and Lindner V: Collagen triple helix repeat containing 1, a novel secreted protein in injured and diseased arteries, inhibits collagen expression and promotes cell migration. Circ Res 96: 261-268, 2005.

21. Ni S, Ren F, Xu M, Tan C, Weng W, Huang Z, Sheng W and Huang D: CTHRC1 overexpression predicts poor survival and enhances epithelial-mesenchymal transition in colorectal cancer. Cancer Med 7: 5643-5654, 2018.

22. Kim HC, Kim YS, Oh HW, Kim K, Oh SS, Kim JT, Kim BY, Lee SJ, Choe YK, Kim DH, et al: Collagen triple helix repeat containing 1 (CTHRC1) acts via ERK-dependent induction of MMP9 to promote invasion of colorectal cancer cells. Oncotarget 5: 519-529, 2014.

23. Tang L, Dai DL, Su M, Martinka M, Li G and Zhou Y: Aberrant expression of collagen triple helix repeat containing 1 in human solid cancers. Clin Cancer Res 12: 3716-3722, 2006.

24. Zhang G and Li G: Novel multiple markers to distinguish melanoma from dysplastic nevi. PLoS One 7: e45037, 2012.

25. Liu G, Sengupta PK, Jamal B, Yang HY, Bouchie MP, Lindner V, Varelas X and Kukuruzinska MA: N-glycosylation induces the CTHRC1 protein and drives oral cancer cell migration. J Biol Chem 288: 20217-20227, 2013.

26. Park EH, Kim S, Jo JY, Kim SJ, Hwang Y, Kim JM, Song SY, Lee DK and Koh SS: Collagen triple helix repeat containing-1 promotes pancreatic cancer progression by regulating migration and adhesion of tumor cells. Carcinogenesis 34: 694-702, 2013.

27. Chen YL, Wang TH, Hsu HC, Yuan RH and Jeng YM: Overexpression of CTHRC1 in hepatocellular carcinoma promotes tumor invasion and predicts poor prognosis. PLoS One 8: e70324, 2013. 
28. Erhardt W, Hebestedt A, Aschenbrenner G, Pichotka B and Blümel G: A comparative study with various anesthetics in mice (Pentobarbitone, Ketamine-Xylazine, Carfentanyl-Etomidate) Res Exp Med (Berl) 184: 159-169, 1984

29. Aghajani M, Mansoori B, Mohammadi A, Asadzadeh Z and Baradaran B: New emerging roles of CD133 in cancer stem cell: Signaling pathway and miRNA regulation. J Cell Physiol 234 21642-21661, 2019.

30. Lim SH, Jang J, Park JO, Kim KM, Kim ST, Park YS, Lee J and Kim HC: CD133-positive tumor cell content is a predictor of early recurrence in colorectal cancer. J Gastrointest Oncol 5: 447-456, 2014

31. Kang M, Kim S and Ko J: Roles of CD133 in microvesicle formation and oncoprotein trafficking in colon cancer. FASEB J 33: 4248-4260, 2019.

32. Diaz-Moralli S, Tarrado-Castellarnau M, Miranda A and Cascante M: Targeting cell cycle regulation in cancer. Pharmaco Ther 138: 255-271, 2013.

33. McKay JA, Douglas JJ, Ross VG, Curran S, Murray GI, Cassidy J and McLeod HL: Cyclin D1 protein expression and gene polymorphism in colorectal cancer. Aberdeen colorectal initiative. Int J Cancer 88: 77-81, 2000.

34. Qie S and Diehl JA: Cyclin D1, cancer progression, and opportunities in cancer treatment. J Mol Med (Berl) 94: 1313-1326, 2016.

35. Diehl JA, Cheng M, Roussel MF and Sherr CJ: Glycogen synthase kinase-3beta regulates cyclin D1 proteolysis and subcellular localization. Genes Dev 12: 3499-3511, 1998.

36. Zhang Y, Zhuang Z, Meng Q, Jiao Y, Xu J and Fan S: Polydatin inhibits growth of lung cancer cells by inducing apoptosis and causing cell cycle arrest. Oncol Lett 7: 295-301, 2014

37. Plati J, Bucur O and Khosravi-Far R: Apoptotic cell signaling in cancer progression and therapy. Integr Biol (Camb) 3: 279-296, 2011.

38. Liu F, Wang B, Wang J, Ling X, Li Q, Meng W and Ma J: Oxymatrine inhibits proliferation and migration while inducing apoptosis in human glioblastoma cells. Biomed Res Int 2016 : 1784161,2016

39. Chen Y, Zhao Z, Chen Y, Lv Z, Ding X, Wang R, Xiao H, Hou C, Shen B, Feng J, et al: An epithelial-to-mesenchyma transition-inducing potential of granulocyte macrophage colony-stimulating factor in colon cancer. Sci Rep 7: 8265, 2017.

40. Kim BR, Kang MH, Kim JL, Na YJ, Park SH, Lee SI, Kang S, Joung SY, Lee SY, Lee DH, et al: RUNX3 inhibits the metastasis and angiogenesis of colorectal cancer. Oncol Rep 36: 2601-2608, 2016.

41. Zucker S and Vacirca J: Role of matrix metalloproteinases (MMPs) in colorectal cancer. Cancer Metastasis Rev 23: 101-117, 2004.

42. Zhu J and Thompson CB: Metabolic regulation of cell growth and proliferation. Nat Rev Mol Cell Biol 20: 436-450, 2019.

43. Chen Y, Liu P, Sun P, Jiang J, Zhu Y, Dong T, Cui Y, Tian Y, An T, Zhang J, et al: Oncogenic MSH6-CXCR4-TGFB1 feedback loop: A novel therapeutic target of photothermal therapy in glioblastoma multiforme. Theranostics 9: 1453-1473, 2019.

44. Tanaka S, Kobayashi W, Haraguchi M, Ishihata K, Nakamura N and Ozawa M: Snail1 expression in human colon cancer DLD-1 cells confers invasive properties without $\mathrm{N}$-cadherin expression. Biochem Biophys Rep 8: 120-126, 2016.

45. Chen JB, Zhu YW, Guo X, Yu C, Liu PH, Li C, Hu J, Li HH, Liu LF, Chen MF, et al: Microarray expression profiles analysis revealed lncRNA OXCT1-AS1 promoted bladder cancer cell aggressiveness via miR-455-5p/JAK1 signaling. J Cell Physiol 234: 13592-13601, 2019.

46. Zheng R, Liang J, Lu J, Li S, Zhang G, Wang X, Liu M, Wang W, Chu H, Tao G, et al: Genome-wide long non-coding RNAs identified a panel of novel plasma biomarkers for gastric cancer diagnosis. Gastric Cancer 22: 731-741, 2019.
47. Yin L, Chen Y, Zhou Y, Deng G, Han Y, Guo C, Li Y,Zeng S and Shen H: Increased long noncoding RNA LASP1-AS is critical for hepatocellular carcinoma tumorigenesis via upregulating LASP1. J Cell Physiol 234: 13493-13509, 2019.

48. Li YJ, Wang Y and Wang YY: MicroRNA-99b suppresses human cervical cancer cell activity by inhibiting the PI3K/AKT/mTOR signaling pathway. J Cell Physiol 234: 9577-9591, 2019.

49. Yu X, Yuan Z, Yang Z, Chen D, Kim T, Cui Y, Luo Q, Liu Z, Yang Z, Fan X, et al: The novel long noncoding RNA u50535 promotes colorectal cancer growth and metastasis by regulating CCL20. Cell Death Dis 9: 751, 2018.

50. Li Y, Zhang H, Zhao Y, Wang C, Cheng Z, Tang L, Gao Y, Liu F, $\mathrm{Li} \mathrm{J}, \mathrm{Li} \mathrm{Y}$, et al: A mandatory role of nuclear PAK4-LIFR axis in breast-to-bone metastasis of ER $\alpha$-positive breast cancer cells Oncogene 38: 808-821, 2019.

51. Du ZH, Bi FF, Wang L and Yang Q: Next-generation sequencing unravels extensive genetic alteration in recurrent ovarian cancer and unique genetic changes in drug-resistant recurrent ovarian cancer. Mol Genet Genomic Med 6: 638-647, 2018

52. Koveitypour Z, Panahi F, Vakilian M, Peymani M, Seyed Forootan F, Nasr Esfahani MH and Ghaedi K: Signaling pathways involved in colorectal cancer progression. Cell Biosci 9: 97, 2019.

53. Suman S, Kurisetty V, Das TP, Vadodkar A, Ramos G, Lakshmanaswamy R and Damodaran C: Activation of AKT signaling promotes epithelial-mesenchymal transition and tumor growth in colorectal cancer cells. Mol Carcinog 53 (Suppl 1): E151-E160, 2014

54. Yang X, Zheng YT and Rong W: Sevoflurane induces apoptosis and inhibits the growth and motility of colon cancer in vitro and in vivo via inactivating Ras/Raf/MEK/ERK signaling. Life Sci 239: 116916, 2019.

55. Zhao GX, Ying YX, Weng SQ, Zhang S, Chen Y, Shen XZ, Dong L and Chen S: CAPS1 promotes colorectal cancer metastasis via snail mediated epithelial mesenchymal transformation. Oncogene 38: 4574-4589, 2019.

56. Zhu D, Zhou J, Zhao J, Jiang G, Zhang X, Zhang Y and Dong M: ZC3H13 suppresses colorectal cancer proliferation and invasion via inactivating Ras-ERK signaling. J Cell Physiol 234: 8899-8907, 2019.

57. Zheng M, Zhou Q, Liu X, Wang C and Liu G: CTHRC1 overexpression promotes cervical carcinoma progression by activating the Wnt/PCP signaling pathway. Oncol Rep 41: 1531-1538, 2019

58. Wang Y, Lee M, Yu G, Lee H, Han X and Kim D: CTHRC1 activates pro-tumorigenic signaling pathways in hepatocellular carcinoma. Oncotarget 8: 105238-105250, 2017.

59. Liu J, Li W, Liu S, Zheng X, Shi L, Zhang W and Yang H: Knockdown of collagen triple helix repeat containing 1 (CTHRC1) inhibits epithelial-mesenchymal transition and cellular migration in glioblastoma cells. Oncol Res 25: 225-232, 2017.

60. Gu L, Liu L, Zhong L, Bai Y, Sui H, Wei X, Zhang W, Huang P, Gao D, Kong Y and Lou G: Cthrcl overexpression is an independent prognostic marker in gastric cancer. Hum Pathol 45: 1031-1038, 2014.

61. Hou M, Cheng Z, Shen H, He S, Li Y, Pan Y, Feng C, Chen X, Zhang Y, Lin M, et al: High expression of CTHRC1 promotes EMT of epithelial ovarian cancer (EOC) and is associated with poor prognosis. Oncotarget 6: 35813-35829, 2015.

62. Abdifetah $\mathrm{O}$ and Na-Bangchang K: Pharmacokinetic studies of nanoparticles as a delivery system for conventional drugs and herb-derived compounds for cancer therapy: A systematic review. Int J Nanomedicine 14: 5659-5677, 2019.

63. Li C, Cai G, Song D, Gao R, Teng P, Zhou L, Ji Q, Sui H, Cai J, $\mathrm{Li} \mathrm{Q}$ and Wang Y: Development of EGFR-targeted evodiamine nanoparticles for the treatment of colorectal cancer. Biomater Sci 7: 3627-3639, 2019.

This work is licensed under a Creative Commons Attribution-NonCommercial-NoDerivatives 4.0 International (CC BY-NC-ND 4.0) License. 